\title{
$N$-Body Simulation of Planetesimal Formation through Gravitational Instability of a Dust Layer in Laminar Gas Disk
}

\author{
Shugo Michikoshi ${ }^{1}$, Eiichiro Kokubo ${ }^{1,2}$, and Shu-ichiro Inutsuka ${ }^{3}$ \\ michikoshi@cfca.jp, kokubo@th.nao.ac.jp, and inutsuka@nagoya-u.jp
}

\begin{abstract}
We investigate the formation process of planetesimals from the dust layer by the gravitational instability in the gas disk using local $N$-body simulations. The gas is modeled as a background laminar flow. We study the formation process of planetesimals and its dependence on the strength of the gas drag. Our simulation results show that the formation process is divided into three stages qualitatively: the formation of wake-like density structures, the creation of planetesimal seeds, and their collisional growth. The linear analysis of the dissipative gravitational instability shows that the dust layer is secularly unstable although Toomre's $Q$ value is larger than unity. However, in the initial stage, the growth time of the gravitational instability is longer than that of the dust sedimentation and the decrease in the velocity dispersion. Thus, the velocity dispersion decreases and the disk shrinks vertically. As the velocity dispersion becomes sufficiently small, the gravitational instability finally becomes dominant. Then wake-like density structures are formed by the gravitational instability. These structures fragment into planetesimal seeds. The seeds grow rapidly owing to mutual collisions.
\end{abstract}

Subject headings: gravitation, instabilities, methods: $n$-body simulations, planets and satellites:formation

\section{Introduction}

In the standard scenario of planet formation, planetesimals are the precursors of planets. Their formation process is one of the unsolved problems of the planet formation theory. Beginning with micron-sized dust grains, they grow to centimeter size in a protoplanetary disk via collisional agglomeration (Weidenschilling 1980; Blum \& Wurm 2000). The least understood growth phase is

\footnotetext{
${ }^{1}$ Center for Computational Astrophysics, National Astronomical Observatory of Japan, Osawa, Mitaka, Tokyo 181-8588, Japan

${ }^{2}$ Division of Theoretical Astronomy, National Astronomical Observatory of Japan, Osawa, Mitaka, Tokyo 1818588, Japan

${ }^{3}$ Department of Physics, Graduate School of Science, Nagoya University, Furo-cho, Chikusa-ku, Nagoya, Aichi 464-8602, Japan
} 
that of growth from centimeter size to kilometer size. Since the gas drag of meter-sized aggregates is weak, they are slightly decoupled from gas with the sub-Keplerian velocity. The resulting headwind decreases their angular momentum and causes inward drift, whose timescale is about a few hundred years (Adachi et al. 1976; Weidenschilling 1977). The growth process in this stage must be faster than this drift timescale. Another problem is the sticking probability of the aggregates. In this stage, since the threshold velocity for the destruction is low, their collisions may lead to destruction (e.g., Blum \& Wurm 2000; Sirono 2004).

A very thin and dense layer of settled dust aggregates in the mid-plane of the protoplanetary disk is gravitationally unstable. Then the gravitational collapse of the dust layer occurs, and kilometer-sized planetesimals are formed directly (Safronov 1969; Goldreich \& Ward 1973). This scenario has the advantage of a very rapid formation timescale, which is on the order of the Keplerian time, thus avoiding the migration of meter-sized aggregates. However, turbulence in the protoplanetary disk may prevent dust from settling to the mid-plane. As dust settles in the protoplanetary disk, a vertical shear velocity develops because the dust-rich gas in the mid-plane rotates with the velocity closer to the Keplerian velocity than that of the dust-depleted gas, which may cause turbulence. The turbulence can mix dust with gas and prevent the dust layer from settling into a dense enough sheet for gravitational instability (Weidenschilling 1980). Problems concerning the gravitational instability of the dust layer still remain unsolved.

There are two methods for calculating the dynamics of dust particles in gas: fluid simulation and $N$-body simulation. One can consider the particles as virtually fluid and treat them with the Euler equation or Navier-Stokes equation. For example, Yamoto \& Sekiya (2006) performed the two-dimensional numerical simulation to investigate the density evolution of the dust layer due to gravitational instability. They assumed that the dust layer is axisymmetric with respect to the rotational axis. They treated the dust component as a pressure-free fluid because the velocity dispersion of dust is negligible if the stopping time $t_{\text {stop }}$ is sufficiently short, where the stopping time $t_{\text {stop }}$ is the characteristic timescale for a particle to stop in gas. They found that the dust layer becomes extremely thin if the stopping time is long. Wakita \& Sekiva (2008) performed the numerical simulation of the gravitational instability of a two-dimensional thin disk and investigated the non-axisymmetric modes.

On the other hand, we can investigate the dynamics of particles in gas by using $N$-body simulations (Tanga et al. 2004; Michikoshi et al. 2007, 2009; Rein et al. 2010). This is straightforward and precise, but the calculation is very time-consuming and the practical number of particles is limited. Tanga et al. (2004) performed $N$-body simulations of a gravitationally unstable disk with a localshearing box. They investigated the formation of clumps of planetesimals at t30 AU by the gravitational instability. They considered the drag force from the background gas. They showed that the planetesimal clumps form owing to the gravitational instability, which correspond to planetesimals in our calculation. In their simulation, the optical depth is smaller than that in our calculation and the particle disk is unstable even initially. We consider the formation of planetesimals at 1 AU, thus we use the large optical depth, and assume the dust layer is initially stable. Michikoshi et al. 
(2007) performed a set of local simulations of the self-gravitating collisional particle disks without gas using $N$-body simulation (hereafter Paper I). Dust particle dynamics is calculated with the Hill equations in a local-shearing box (Wisdom \& Tremaine 1988). They adopted the rubble pile model (hard and soft sphere models) as collision models. They found that the formation process is divided into three stages: the formation of non-axisymmetric wake-like structures (Salo 1995), the creation of aggregates, and the rapid collisional growth of the aggregates. The mass of the largest aggregates is larger than the mass predicted by the linear perturbation theory. Michikoshi et al. (2009) adopted the alternative model of collisions, 'accretion model' (Paper II). In the accretion model, the number of particles decreases as the calculation proceeds; thereby this model enables us to perform large-scale and long-term simulations. They obtained the final mass of planetesimals as a function of the size of the computational box. Rein et al. (2010) performed the numerical simulations to investigate the validity of the super-particle approximation. They considered the various physics, such as the gas drag, the self-gravity, the physical collision and the turbulence. They treated the collisions as the hard sphere model. They investigated the numerical requirements to study the gravitational instability.

To understand the basic dynamics of gravitational instability, we neglected the effect of gas in Papers I and II. However, it is obvious that the gas plays an important role in planetesimal formation when dust particles are small. The interaction with gas through drag is dominant in the dynamical evolution of particles. Many authors investigated the effect of gas. The particles drift radially due to gas drag (Adachi et al. 1976; Weidenschilling 1977; Nakagawa et al. 1986). As the sedimentation of dust aggregates towards the midplane proceeds, the vertical velocity shear increases. Thus, the Kelvin-Helmholtz instability occurs (Weidenschilling 1980; Cuzzi et al. 1993; Sekiva 1998; Dobrovolskis et al. 1999; Sekiva \& Ishitsu 2000, 2001; Ishitsu \& Sekiva 2002, 2003; Gómez \& Ostriker 2005; Michikoshi \& Inutsuka 2006; Johansen et al. 2006; Chiang 2008; Barranco 2009). The Kelvin-Helmholtz instability makes the dust layer turbulent. The turbulent gas prevents the concentration of dust (Weidenschilling \& Cuzzi 1993), and helps the concentration (Barge \& Sommeria 1995; Fromang \& Nelson 2006). The interaction between gas and dust causes the streaming instability (Youdin \& Goodman 2005). By the streaming instability, the dust grains concentrate strongly and the large Ceres-sized planetesimals form (Youdin \& Johansen 2007; Johansen \& Youdin 2007; Johansen et al. 2007, 2009). The loss of angular momentum due to the gas drag helps gravitational instability (Ward 1976; Youdin 2005a, b). The time-scale for the dissipative instability is relatively slow, but the dust layer may be unstable even when the Toomre's Q value is larger than unity. As a first step toward understanding the effect of gas on gravitational instability, we introduce gas as a background laminar flow in the present paper. The aim of the present paper is to investigate the effect of the laminar gas on the planetesimal formation through gravitational instability. The laminar flow causes the dissipation of the kinetic energy and thus radial migration of dust.

In $\S 2$, we summarize the results of the linear analysis of the gravitational instability under gas drag. In $\S 3$, we describe the simulation method and the initial condition. In $\S 4$, we present our 
numerical results. $\S 5$ is devoted to discussions. We summarize the results in $\S 6$.

\section{Dispersion Relation for Gravitational Instability under Gas Drag}

The linear stability analyses of the dust layer with a finite thickness were performed by Sekiva (1983) and Yamoto \& Sekiya (2004). They assumed that the size of dust aggregates is sufficiently small, in other words, dust is fully coupled with gas, thus they treated gas and dust as one fluid. That introduces no dissipation due to gas drag. They considered the vertical structure of the dust layer, and studied the Roche criterion. The linear stability analyses of dissipative gravitational instability were performed by Ward (1976), Coradini et al. (1981), and Youdin (2005a). In this section, we summarize the essence of their results. Ward (1976) and Youdin (2005a) used one component model, i.e., they neglected the dynamics of gas flow, but they consider the gas drag from the stationary background gas flow. Using their results, we discuss conditions for the gravitational instability under the influence of gas drag.

\subsection{Dispersion Relation}

We adopt the isothermal equation of state for the dust layer:

$$
p=c^{2} \rho,
$$

where $p$ is the pressure, $c$ is the isothermal sound speed, and $\rho$ is the density of the dust layer. In this equation of state, the velocity dispersion of dust is always constant. Clearly, the velocity dispersion must not be constant. It changes owing to several processes such as gas drag, inelastic collisions, and gravitational scattering. This assumption gives us the simple analytical expression of the dispersion relation, with which we can grasp the nature of dissipative gravitational instability.

We assume that the gas velocity is equal to the Kepler velocity. We consider the reference point that is at the semi-major axis $a_{0}$. The Kepler angular velocity of the reference point is $\Omega=\sqrt{G M_{\mathrm{s}} / a_{0}^{3}}$ where $M_{\mathrm{s}}$ is the mass of the central star and $G$ is the gravitational constant. We introduce the local Cartesian coordinates in which the $x$-axis is directed radially, the $y$-axis follows the direction of rotation, and the $z$-axis follows the direction perpendicular to mid-plane. We define $\mathbf{v}=\left(v_{x}, v_{y}, v_{z}\right)$ as the deviation velocity field from the local Kepler velocity.

The basic equations for the dust layer in this frame are given by

$$
\begin{gathered}
\frac{\partial \rho}{\partial t}+\nabla \cdot(\rho \mathbf{v})=\frac{3}{2} \Omega x \frac{\partial \rho}{\partial y} \\
\frac{\partial \mathbf{v}}{\partial t}+(\mathbf{v} \cdot \nabla) \mathbf{v}=-\frac{\nabla p}{\rho}+\left(2 v_{y} \Omega,-\frac{1}{2} v_{x} \Omega,-z \Omega^{2}\right)+\frac{3}{2} \Omega x \frac{\partial \mathbf{v}}{\partial y}-\frac{1}{t_{\text {stop }}} \mathbf{v}-\nabla \phi \\
\nabla^{2} \phi=4 \pi G \rho,
\end{gathered}
$$


where $\phi$ is the gravitational potential due to the self-gravity. The simulation results in $\S 4$ show that the density fluctuation is not axisymmetric. However, for the sake of simplicity, restricting ourselves to the axisymmetric mode $(\partial / \partial y=0)$, we carry out a normal mode analysis in the form of $\exp (-i(\omega t-k x))$ where $k$ is the wave number in the $x$-direction and $\omega$ is the growth rate.

Integrating toward $z$, we obtain the equation of perturbed quantities $\Sigma_{1}, v_{x 1}, v_{y 1}, \phi_{1}$ :

$$
\begin{gathered}
-i \omega \Sigma_{1}+i k \Sigma_{0} v_{x 1}=0 \\
-i \omega v_{x 1}=2 \Omega v_{y 1}-i k c^{2} \frac{\Sigma_{1}}{\Sigma_{0}}+\frac{1}{t_{\text {stop }}} v_{x 1}-i k \phi_{1}, \\
-i \omega v_{y 1}=-\frac{1}{2} \Omega v_{x 1}-\frac{1}{t_{\text {stop }}} v_{y 1}, \\
-2 \pi G \Sigma_{1}=k \phi_{1},
\end{gathered}
$$

where $\Sigma$ is the surface density of the dust layer. Youdin (2005a) considered the finite thickness model using the softening term (Vandervoort 1970). Poisson equation with the softening parameter is

$$
-2 \pi G \Sigma_{1} T(k h)=k \phi_{1},
$$

where $h$ is the scale height of the dust layer and $T(k h)$ is the softening parameter:

$$
T(k h)=\frac{1}{1+k h} .
$$

For nontrivial solutions, the determinant of the coefficient matrix must vanish. Thus the following dispersion relation is obtained (Youdin 2005a):

$$
\mu^{3}+\frac{2}{t_{\text {stop }}} \mu^{2}+\left(\Omega^{2}+\frac{1}{t_{\text {stop }}^{2}}-2 \pi k G \Sigma_{0} T(k h)+c^{2} k^{2}\right) \mu+\frac{c^{2} k^{2}-2 \pi k \Sigma_{0} G}{t_{\text {stop }}}=0,
$$

where $\mu=-i \omega$. The mode for $\Re(\mu)>0$ is unstable, where $\Re(X)$ is the real part of a complex number $X$. In the gas-free limit $\left(t_{\text {stop }} \rightarrow \infty\right)$ and the thin disk limit $(k h \rightarrow 0)$, the dispersion relation converges to the conventional dispersion relation of a thin disk (Toomre 1964; Goldreich \& Ward 1973). Equation (11) can be written in a non-dimensional form scaled by the length $c / \Omega$ and the time $\Omega^{-1}$ :

$$
\tilde{\mu}^{3}+\frac{2}{\tilde{t}_{\text {stop }}} \tilde{\mu}^{2}+\left(\frac{1}{\tilde{t}_{\text {stop }}^{2}}+\tilde{k}^{2}+1-\frac{2 \tilde{k}}{Q} T(\tilde{k} \tilde{h})\right) \tilde{\mu}+\frac{\tilde{k}^{2} Q-2 \tilde{k}}{\tilde{t}_{\text {stop }} Q}=0,
$$

where we used Toomre's $Q$ given by $Q=\Omega c /\left(\pi G \Sigma_{0}\right)$. A tilde denotes non-dimensional quantity hereafter.

A dissipative dust layer is secularly unstable for long-wavelength modes although $Q>1$. The physical mechanism of the instability is explained as follows (Goodman \& Pindor 2000; Chiang \& Youdin 2010). We consider a thin axisymmetric overdense ring and the thick back-ground gas. From the 
force balance of the self-gravity, we find that the dust rotates faster at the outer edge of the ring. Thus, the drag force at the outer edge is strong. This causes the inward drift. Conversely, the dust at the inner edge drifts outward. These effects shrink ring radially.

Figure 1 shows the dispersion relation of the dust layer under gas drag for $Q=0.5$. We used the thin disk model $(h=0)$. The stopping time is $0.2,1.0,5.0$, and $\infty$ (gas-free). All models have the maximum growth rate at $\tilde{k}=2$. We will see that the growth rate has the maximum value at $\tilde{k}=1 / Q$ in $\$ 2.2$. As the stopping time lengthens, the growth rate shrinks. In the gas-free model, long-wavelength modes are stable, while in the gas model, they are unstable.

\subsection{Growth Rate of the Most Unstable Mode}

As discussed in $\$ 2.1$, the dust layer is always unstable for long-wavelength modes. However, the timescale of the instability can be longer than the dynamical timescale, because this is the secular instability. Therefore, in order to understand what happens, we have to compare the timescale of the instability with those of other processes such as the sedimentation, the radial migration, and the growth of dust aggregates.

Here, we assume the thin disk approximation $(h=0)$ in order to obtain the simple analytical formula. For $\tilde{k}<2 / Q, \tilde{\mu}$ is a real positive value. The growth rate for the most unstable mode $\tilde{\mu}$ is a function of $\tilde{k}, \tilde{t}_{\text {stop }}$, and $Q$. From Equation (12), the partial derivative of $\tilde{\mu}$ with respect to $\tilde{k}$ is given as

$$
\frac{\partial \tilde{\mu}}{\partial \tilde{k}}=\frac{2(1 / Q-\tilde{k})(\tilde{\mu}+1)}{4 \tilde{\mu} / \tilde{t}_{\text {stop }}+1 / \tilde{t}_{\text {stop }}^{2}+\tilde{k}^{2}+1-2 \tilde{k} / Q+3 \tilde{\mu}^{2}} .
$$

Using Equation (12), we can show that the denominator on the right hand side of Equation (13) is positive when $\tilde{k}<2 / Q$. Hence, the $\tilde{\mu}$ has the maximum value at $\tilde{k}=1 / Q$ for a fixed $\tilde{t}_{\text {stop }}$ and $Q$. The substitution of $\tilde{k}=1 / Q$ into Equation (12) provides the equation of the maximum growth rate $\tilde{\mu}_{\max }$ :

$$
\tilde{\mu}_{\text {max }}^{3}+\frac{2}{\tilde{t}_{\text {stop }}} \tilde{\mu}_{\text {max }}^{2}+\left(\frac{1}{\tilde{t}_{\text {stop }}^{2}}+1-\frac{1}{Q^{2}}\right) \tilde{\mu}_{\text {max }}-\frac{1}{\tilde{t}_{\text {stop }} Q^{2}}=0 .
$$

To obtain the condition that the gravitational instability dominates over the other process (symbolically denoted by "x"), we equate $\tilde{T}_{X}$ with $\tilde{\mu}_{\max }^{-1}$ where $T_{X}$ is the characteristic timescale of a process $\mathrm{X}$, ans solve the resultant equation with respect to $\mathrm{Q}$ to obtain $Q$ value:

$$
Q_{\text {crit }, \mathrm{X}}=\tilde{T}_{X} \sqrt{\frac{\tilde{t}_{\text {stop }}\left(\tilde{T}_{X}+\tilde{t}_{\text {stop }}\right)}{\tilde{t}_{\text {stop }}^{2} \tilde{T}_{X}^{2}+\left(\tilde{T}_{X}+\tilde{t}_{\text {stop }}\right)^{2}}} .
$$

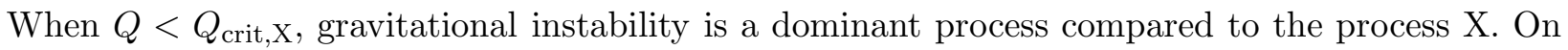
the other hand, when $Q>Q_{\text {crit, } \mathrm{X}}$, the timescale for the process $\mathrm{X}$ is shorter than the timescale of gravitational instability. Although the dust layer is unstable, the process $\mathrm{X}$ is dominant. 


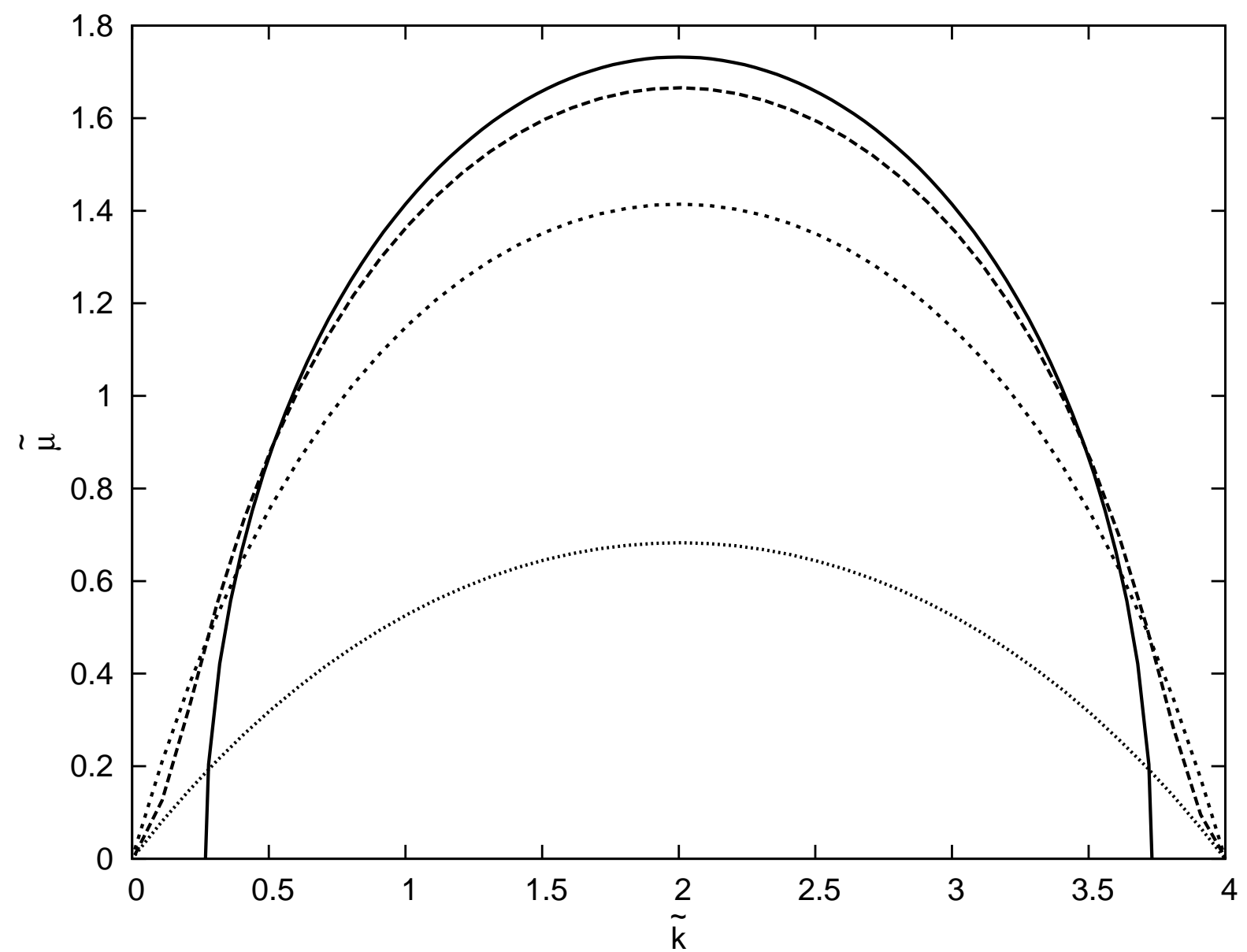

Fig. 1. - The dispersion relation of the dust layer under gas drag for $Q=0.5$. We used the thin disk model $(h=0)$. The stopping time is 0.2 (dotted curve), 1.0 (short-dashed curve), and 2.0 (dashed curve). The solid curve denotes the dispersion relation for the gas-free model. 
We can derive the leading term of the power series expansion with respect to $t_{\text {stop }}$ for Equation (13) and find the asymptotic solution (Ward 1976; Youdin 2005a; Chiang \& Youdin 2010). When the gas drag is strong $\tilde{t}_{\text {stop }} \ll 1$, the growth rate for the most unstable mode is

$$
\tilde{\mu}_{\max }=\frac{\tilde{t}_{\text {stop }}}{Q^{2}} .
$$

Conversely, when the gas drag force is weak $t_{\text {stop }} \gg 1$ and $Q>1$, the growth rate for the most unstable mode is

$$
\tilde{\mu}_{\max }=\frac{1}{\left(Q^{2}-1\right) \tilde{t}_{\text {stop }}} .
$$

When $Q<1$, the growth rate for the most unstable mode converges to the finite value $\sqrt{1 / Q^{2}-1}$ as $\tilde{t}_{\text {stop }} \rightarrow \infty$, which is the maximum growth rate for the gas-free gravitational instability.

\section{Method of Calculation}

\subsection{Model}

The method of calculation is the same as those used in Papers I and II except for including gas, which is based on the method of the local simulation of planetary rings (e.g., Wisdom \& Tremaine 1988; Richardson 1994; Daisaka \& Ida 1999).

We describe quantities in the non-dimensional form independent of the semi-major axis $a_{0}$, and the mass of the central star $M_{\mathrm{s}}$ by scaling the time by $\Omega_{0}^{-1}$, the length by the Hill radius $\tilde{h} a_{0}=r_{\mathrm{H}}$, and the mass by $\tilde{h}^{3} M_{\mathrm{s}}$, where $\tilde{h}=\left(2 m_{\mathrm{p}} / 3 M_{\mathrm{s}}\right)^{1 / 3}$ and $m_{\mathrm{p}}$ is the initial mass of particles (Petit \& Henon 1986; Nakazawa \& Ida 1988). The initial mass of particles $m_{\mathrm{p}}$ is assumed to be identical. The non-dimensional Hill equations for the particle $i$ are given by (e.g., Nakazawa \& Ida 1988):

$$
\begin{aligned}
\frac{d^{2} \tilde{x}_{i}}{d \tilde{t}^{2}} & =2 \frac{d \tilde{y}_{i}}{d \tilde{t}}+3 x_{i}+\sum_{j} \frac{\tilde{m}_{j}}{\tilde{r}_{i j}^{3}}\left(\tilde{x}_{j}-\tilde{x}_{i}\right)-\frac{1}{\tilde{t}_{\mathrm{stop}, i}}\left(\frac{d \tilde{x}_{i}}{d \tilde{t}}-\tilde{v}_{\mathrm{g} x}\right), \\
\frac{d^{2} \tilde{y}_{i}}{d \tilde{t}^{2}} & =-2 \frac{d \tilde{x}_{i}}{d \tilde{t}}+\sum_{j} \frac{\tilde{m}_{j}}{\tilde{r}_{i j}^{3}}\left(\tilde{y}_{j}-\tilde{y}_{i}\right)-\frac{1}{\tilde{t}_{\mathrm{stop}, i}}\left(\frac{d \tilde{y}_{i}}{d \tilde{t}}-\tilde{v}_{\mathrm{g} y}\right), \\
\frac{d^{2} \tilde{z}_{i}}{d \tilde{t}^{2}} & =-\tilde{z}_{i}+\sum_{j} \frac{\tilde{m}_{j}}{\tilde{r}_{i j}^{3}}\left(\tilde{z}_{j}-\tilde{z}_{i}\right)-\frac{1}{\tilde{t}_{\mathrm{stop}, i}}\left(\frac{d \tilde{z}_{i}}{d \tilde{t}}-\tilde{v}_{\mathrm{g} z}\right),
\end{aligned}
$$

where $\left(\tilde{x}_{i}, \tilde{y}_{i}, \tilde{z}_{i}\right), \tilde{m}_{i}$, and $\tilde{t}_{\mathrm{stop}, i}$ are the position, mass, and the stopping time of the particle $i, \tilde{r}_{i j}$ is the distance between particles $i$ and $j$, and $\left(\tilde{v}_{\mathrm{g} x}, \tilde{v}_{\mathrm{g} y}, \tilde{v}_{\mathrm{g} z}\right)$ is the velocity field of gas.

We neglect the dust back-reaction on gas for the sake of simplicity. We assume that the laminar gas flow, which has the sub-Keplerian velocity field:

$$
\left(\tilde{v}_{\mathrm{g} x}, \tilde{v}_{\mathrm{g} y}, \tilde{v}_{\mathrm{g} z}\right)=\left(0,-\frac{3}{2} \tilde{x}-\tilde{v}_{\mathrm{dif}}, 0\right)
$$


where $-3 \tilde{x} / 2$ is the Keplerian shear velocity, $\tilde{v}_{\text {dif }}$ is the difference from the Keplerian velocity. The stopping time $\tilde{t}_{\mathrm{stop}, i}$ depends on the size of particle: $\tilde{t}_{\mathrm{stop}, i}=\tilde{t}_{\mathrm{stop}, 0}\left(\tilde{m}_{i} / \tilde{m}_{p}\right)^{\alpha}$ where $\alpha$ is the powerlaw index, and $\tilde{t}_{\mathrm{stop}, 0}$ is the stopping time of the particle with $\tilde{m}_{\mathrm{p}}$. In this paper, we adopt $\alpha=0$ and 2/3. The index $\alpha=2 / 3$ corresponds to Stokes' law (e.g., Landau \& Lifshitz 1959).

The calculation box has the periodic boundary condition (e.g., Wisdom \& Tremaine 1988). The size of the box is taken as a square $A \lambda_{\mathrm{m}} \times A \lambda_{\mathrm{m}}$ where $\lambda_{\mathrm{m}}$ is the most unstable wavelength of gravitational instability and $A$ is the non-dimensional parameter.

The system is characterized by two non-dimensional parameters, the optical depth and the ratio of the Hill radius to the diameter of a particle (Daisaka \& Ida 1999):

$$
\begin{gathered}
\tau=\frac{3 \Sigma}{4 \rho_{\mathrm{p}} r_{\mathrm{p}}}, \\
\zeta=\frac{r_{\mathrm{H}}}{2 r_{\mathrm{p}}},
\end{gathered}
$$

where $\rho_{\mathrm{p}}$ and $r_{\mathrm{p}}$ are the internal density and initial radius of the particle, respectively. The realistic value for "solid" dust particles is $\zeta \simeq 105.28$ for $\rho_{\mathrm{p}}=2 \mathrm{~g} / \mathrm{cm}^{3}$ and $a_{0}=1$ AU. However, we adopt $\zeta \simeq 2$ in this paper owing to the computational limit (see Papers I and II). We set the optical depth $\tau=0.1$, which is approximately equal to the realistic value $\tau=0.19$ for $\Sigma_{\mathrm{d}}=10 \mathrm{~g} / \mathrm{cm}^{3}, r_{\mathrm{p}}=20 \mathrm{~cm}$, and $\rho_{\mathrm{p}}=2 \mathrm{~g} / \mathrm{cm}^{3}$.

Since our choice of $\zeta$ is smaller than the realistic value, a particle used in the simulation is not a realistic dust particle but a 'super-particle' that represents a group of many small particles that have the same position and velocity. Thus the restitution coefficient $\epsilon$ corresponds to the rate of the dissipation due to collisions between super-particles. The low value of $\zeta$ means that the physical size of a particle in our simulation is very large. This implies that the effect of the gravitational scattering is relatively weaker than that of collisions. We investigated the $\zeta$ value dependence in paper I and II in the narrow range $(\zeta=1.5-3.0)$ and we confirmed that the formation process does not change in this range qualitatively. However, we show that the collisional growth is important in the final stage where |zeta controls the growth rate. Thus, we should perform the large $\zeta$ value. In the future work, we will concentrate on the collisional growth and perform the $N$-body simulations with a realistic $\zeta$ value.

In the Hill coordinates, the Keplerian orbit is determined by six parameters: the position of the guiding center, eccentricity $e$, inclination $i$, and two phases for epicyclic and vertical oscillations (Nakazawa \& Ida 1988). The initial eccentricity and inclination of particles are assumed to follow the Rayleigh distribution. We fix $\sqrt{\left\langle e^{2}\right\rangle /\left\langle i^{2}\right\rangle}=2$ according to Ida \& Makino (1992).

The other parameters are uniformly distributed, avoiding overlapping.

We adopt the hard-sphere and accretion models as a collision model (Papers I and II). In the hard-sphere model, the penetration of particles is not possible (e.g., paper I, Richardson 1994). When a collision occurs, the particle velocity changes instantly. The relative tangential velocity 
is conserved and the magnitude of the relative normal velocity is reduced by a factor of $\epsilon$. We use the constant $\epsilon$. We also use the accretion model (e.g., Paper II, Kokubo et al. 2000). In this model, when two particles collide, if the binding condition is satisfied, the two particles merge into one particle. The binding condition is described by the Jacobi integral $J<0$ (e.g., Paper II, Nakazawa \& Ida 1988).

In the hard-sphere model, since a planetesimal consists of many small particles, it can be distorted in shape and break up. On the other hand, in the accretion model, a planetesimal cannot be distorted or break up. In this respect, the hard-sphere model is more realistic than the accretion model. However the accretion model has an advantage. First, since the number of particles decreases as the calculation proceeds, this model enables us to reduce the calculation cost. Second, we can easily handle size-dependent drag coefficient. In general, the drag coefficient depends on the size of a particle. In the hard sphere model, it is complicated to handle the sizedependent drag coefficient. In the accretion model, we can adopt the size-dependent drag without any difficulty. We have shown that results in the accretion model are the same as those in the hard sphere model in the gas-free model (Paper II).

\subsection{Test Simulations}

We compare the accretion model with the hard sphere model in the laminar gas flow. We performed simulations with the same model parameters except for collision models (models 100H0, $100 \mathrm{AC0}$, and 100AS0) in order to check the validity of the accretion model. The initial stopping time of particles is $\tilde{t}_{\text {stop }, 0}=1.0$. In the accretion models, we calculate the drag coefficient using the constant model $(\alpha=0)$ and the Stokes' law model $(\alpha=2 / 3)$. The left panel of Figure 2 shows the time evolution of $Q$. In the phase where $Q$ decreases, there are no clear differences among all models. On the other hand, in the phase where $Q$ increases, the discrepancies appear among them. Toomre's $Q$ value in the accretion model with $\alpha=2 / 3$ is larger than those in the hard sphere model and the accretion model with $\alpha=0$. In the Stokes' law model, as particles grow, the drag coefficient becomes small and thus the dissipation becomes inefficient, which leads to the relatively large velocity dispersion.

The right panel of Figure 2 shows the maximum mass of planetesimals for all models. In the early stage, particles or aggregates do not grow. There the difference in collision models is not important. The difference can be seen in the late stage. In the Stokes' law model $\alpha=2 / 3$, the drag coefficient changes as particles grow, which affects the evolution of the maximum mass. In the hard-sphere model, the drag coefficient is constant although aggregates grow, therefore the evolution in the hard-sphere model must be similar to that in the accretion model with $\alpha=0$. However, the difference appears at $t / t_{\mathrm{K}}=10$. The maximum mass of the accretion model with $\alpha=0$ increases, but that of the hard-sphere model does not change. At $t / t_{\mathrm{K}}=10$, there are only a few particles or aggregates. The maximum mass of planetesimals is sensitive to the initial condition and noise. But, at $t / t_{\mathrm{K}}=20$, the maximum mass converges to the same value. The final mass of 
the planetesimal depends on the size of the computational domain (Michikoshi et al. 2009). The dispersion of the mass of the final state is relatively small.

In the early stage where no planetesimals form, the time evolution is quite similar in all models. In the late stage, once planetesimals form, there are no remarkable differences among all models. If we focus on the the evolution of statistical value, such as the velocity dispersion, the number of planetesimals, we can investigate the gravitational instability and planetesimal formation using the accretion model. Although the time evolution of the maximum mass of the planetesimal in the late stage depends on the initial noise, the maximum mass finally converge to the same value. Therefore, we adopt the accretion model to investigate the gravitational instability from now on.

\section{Results}

We performed 14 simulations with different disk models. The model parameters are summarized in Table 1. We fixed the following parameters: the optical depth, $\tau=0.1$, the restitution coefficient $\epsilon=0.01$, the ratio of the Hill radius to the diameter $\zeta=2.0$, the initial Toomre's $Q$ value $Q_{\text {init }}=3$, and the size of the computational domain $A=6$. The basic dependence of planetesimal formation of these parameters was investigated in Papers I and II.

\subsection{Time evolution}

Figure 3 shows the typical evolution of the simulation (model 100AS0). At $t / t_{\mathrm{K}}=0$, and 0.2 , particles are distributed randomly and uniformly in the computational box. No gravitational instability occurs, thus we can see no remarkable structures. At $t / t_{\mathrm{K}}=0.4$, the large non-axisymmetric density structures appear. Then, the gravitational instability seems to start. In the model where $\left(t_{\text {stop }}=0.25\right)$, the structure is not clear. For the short stopping time models, the velocity dispersion is small and the critical wave length is short. Thus, we cannot observe the large density structure clearly. At $t / t_{\mathrm{K}}=0.6$, particles begin to grow in the dense region. At $t / t_{\mathrm{K}}=0.8$, and 1.0 , particles continue to grow, and the number of small particles decreases rapidly. The small particles are absorbed by large particles. The rapid collisional growth of particles continues until the one large planetesimal finally forms.

The formation process of planetesimals in the laminar gas model is similar to those in the gas-free model (papers I and II). The gas drag from the laminar gas does not qualitatively change formation process. This process is divided into three stages: the formation of density structures, the creation of planetesimal seeds, and their collisional growth.

The linear stability analysis of gas-free gravitational instability shows that the disk is stable when $Q>1$ (Toomre 1964). As discussed in $\S 3$, the dust layer in the laminar gas disk is secularly unstable although $Q>1$. However, the growth time of the dissipative gravitational instability 
Table 1. Simulation parameters

\begin{tabular}{ccccc}
\hline \hline Model & $\tilde{t}_{\text {stop }, 0}$ & Collision & $\alpha$ & $\tilde{v}_{\text {dif }}$ \\
\hline 010AS0 & 0.10 & Accretion & $2 / 3$ & 0 \\
025AS0 & 0.25 & Accretion & $2 / 3$ & 0 \\
050AS0 & 0.50 & Accretion & $2 / 3$ & 0 \\
100AS0 & 1.00 & Accretion & $2 / 3$ & 0 \\
200AS0 & 2.00 & Accretion & $2 / 3$ & 0 \\
400AS0 & 4.00 & Accretion & $2 / 3$ & 0 \\
1000AS0 & 10.00 & Accretion & $2 / 3$ & 0 \\
INFA & $\infty$ & Accretion & - & 0 \\
100AC0 & 1.00 & Accretion & 0 & 0 \\
100H0 & 1.00 & Hard & 0 & 0 \\
100AC1 & 1.00 & Accretion & 0 & 10 \\
100AS1 & 1.00 & Accretion & $2 / 3$ & 10 \\
100AC2 & 1.00 & Accretion & 0 & 20 \\
100AS2 & 1.00 & Accretion & $2 / 3$ & 20 \\
\hline
\end{tabular}

Note. - Parameters $\tilde{t}_{\text {stop }, 0}, \alpha$, and $v_{\text {dif }}$ are the stopping time of particles, the power-law index for the stopping time $\tilde{t}_{\text {stop }, i} \propto \tilde{m}_{i}^{\alpha}$, and the velocity difference from Kepler velocity. We fixed other parameters: the optical depth $\tau=0.1$, the restitution coefficient $\epsilon=0.01$, the ratio of the Hill radius to the diameter $\zeta=2.0$, the initial $Q$ value $Q_{\text {init }}=3$, and the size of the computational domain $A=6$. 
is longer than the sedimentation in the initial stage. Thus, the disk shrinks vertically. As the velocity dispersion and the scale height decrease, the growth rate of the dissipative gravitational instability increases. Then, the gravitational instability becomes a dominant process, and the wake-like structures appear. These structures fragment into the seeds of planetesimals. The seeds grow rapidly owing to mutual collisions. Finally, almost all mass in the computational domain is absorbed by only one planetesimal in this calculation. The size of the final planetesimal depends on the size of the computational domain (paper II).

\section{2. $\quad$ Effect of Stopping Time}

We assume that the difference in the rotational velocity from the Kepler velocity is equal to zero $\left(v_{\text {dif }}=0\right)$ (models INFA, 010AS0 - 1000AS0). In the model INFA, $t_{\text {stop }, 0}=\infty$, i.e., the drag term is neglected. In other models, the stopping times are $\tilde{t}_{\mathrm{stop}, 0}=0.1,0.25,0.5,1.0,2.0,4.0$, and 10.0. We adopt the Stokes' law model, $\alpha=2 / 3$.

\subsubsection{Toomre's $Q$ value}

The top panel of Figure 4 shows the time evolution of Toomre's $Q$ values. In all models, Toomre's $Q$ value initially decreases. In the gas-free model (model INFA), the kinetic energy is dissipated only by inelastic collisions. The dissipation timescale due to inelastic collisions is approximately estimated by $t_{\mathrm{c}} \simeq 1 /\left(\tau \Omega\left(1-\epsilon^{2}\right)\right) \simeq 10.0 \Omega^{-1}$ (Goldreich \& Tremaine 1978). In the laminar gas models, the decrease of Toomre's $Q$ value is caused by the dissipation due to both the gas drag and inelastic collisions.

The top panel of Figure 5 shows the dependence of the decay time $t_{\text {decay, } \mathrm{Q}}$ on $t_{\mathrm{stop}, 0}$, where the decay time of Toomre's $Q$ value $t_{\text {decay, } \mathrm{Q}}$ is defined by

$$
t_{\text {decay }, \mathrm{Q}}=t_{\min , \mathrm{Q}} \frac{Q_{\text {init }}}{Q_{\text {init }}-Q_{\min }},
$$

where $Q_{\min }$ is the minimum $Q$ value at $t=t_{\mathrm{min}, \mathrm{Q}}$. The decay time $t_{\text {decay, } \mathrm{Q}}$ is proportional to $t_{\text {stop }, 0}$ for $\tilde{t}_{\text {stop }, 0}<1$. Toomre's $Q$ value is proportional to the velocity dispersion $\sigma_{x}$. The inelastic collisions and the gas drag decrease the velocity dispersion. If the drag is sufficiently strong, the decay time scale of the velocity dispersion is on the order of the stopping time. The timescale of the decay of the velocity dispersion due to inelastic collisions is about $t_{\mathrm{c}} \simeq 10.0 \Omega^{-1}$. Thus, when

$\tilde{t}_{\text {stop }, 0}>1$ as $\tilde{t}_{\text {stop }, 0}$ increases, we cannot neglect collisions. Therefore the decay timescale of the velocity dispersion is smaller than the stopping time for $\tilde{t}_{\text {stop }, 0}>1$.

In all models, Toomre's $Q$ value has the minimum value $Q_{\min }$ at $t_{\mathrm{min}, \mathrm{Q}}$. In the gas-free model, the minimum $Q$ value is about 2 (papers I, and II), and according to the linear theory, the critical value of a thin disk is $Q=1$. This is because the decrease in the velocity dispersion due to inelastic 
collisions is sufficiently slow. However, as shown in Figure 4, if the gas drag is strong, $Q$ value becomes smaller than unity. As the stopping time shortens, the minimum $Q$ value decreases. For example, the minimum $Q$ value is 0.15 for $\tilde{t}_{\text {stop }, 0}=0.25$.

The axisymmetric structure starts forming when $Q$ value is minimum. For example, in the model where $\tilde{t}_{\mathrm{stop}, 0}=1.0, Q$ value is minimum at $t / t_{\mathrm{K}}=0.3$, and we can see the non-axisymmetric structure at $t / t_{\mathrm{K}}=0.35$. Particles are scattered by the non-axisymmetric structures, and the velocity dispersion of particles increases.

If the decrease of the $Q$ value is more rapid than the gravitational instability, the dust layer cannot collapse although the dust layer is gravitationally unstable. As $Q$ value decreases, the timescale of the gravitational instability becomes short, therefore, the critical $Q$ value is determined by $t_{\mathrm{stop}, 0}=t_{\mathrm{GI}}$, where $t_{\mathrm{GI}}$ is the timescale of the gravitational instability. Here, we assume $t_{\mathrm{stop}, \mathrm{i}}=$ $t_{\text {stop }, 0}$ because particles do not grow much before gravitational instability. From Equation (15), we obtain the following condition:

$$
Q_{\text {min }}=\sqrt{\frac{2 \tilde{t}_{\text {stop }, 0}^{2}}{4+\tilde{t}_{\text {stop }, 0}^{2}}} .
$$

The minimum Toomre's $Q$ value as a function of $t_{\mathrm{stop}, 0}$ is shown in the bottom panel of Figure 5 , Strictly speaking, Equation (25) is valid only when the disk is thin. Nevertheless, the analytical expression agrees with the numerical result.

\subsubsection{Roche Density}

We define the scale height of a dust layer as the mean square root of $z, h=\sqrt{2}\left\langle z_{i}^{2}\right\rangle^{1 / 2}$. We can calculate the mean density of the dust layer from the scale height, $\rho=\Sigma / h$. The density at which the self-gravity is equal to the tidal force is called the Roche density, which is defined by the following equation (e.g., Yamoto \& Sekiya 2004):

$$
\rho_{\mathrm{R}}=\frac{9}{4 \pi} \frac{M_{\mathrm{s}}}{a_{0}^{3}}
$$

The dust layer may collapse if the dust layer density exceeds the Roche density. The linear analysis gives the more precise criterion (Sekiva 1983; Yamoto \& Sekiva 2004). However, the criterion does not change very much. Thus, we introduce $Q_{\mathrm{R}}$ given by

$$
Q_{\mathrm{R}}=\frac{\rho_{\mathrm{R}}}{\rho}=\frac{9}{4 \pi} \frac{h \Omega^{2}}{G \Sigma},
$$

which corresponds to the non-dimensional scale height.

The bottom panel of Figure 4 shows the time evolution of $Q_{R}$. The time evolution of $Q_{R}$ is similar to Toomre's $Q$. The value $Q_{R}$ has the minimum value $h_{\min }$ at $t_{\min , \mathrm{h}}$ in all models. But, the time evolution of $Q_{R}$ is slower than that of $Q$ value. This result indicates that the standard 
hydrostatic relation $h \simeq \sqrt{2} \sigma_{z} / \Omega$ is not satisfied. Figure 6 shows the time evolution of the ratio $h / \sigma_{z}$, which is on the order of the sound crossing time across a scale height. In the gas-free model, the ratio $h / \sigma_{z}$ is nearly constant in $t / t_{\mathrm{K}}<1$. The hydrostatic relation is satisfied. In a laminar gas model , the ratio $h / \sigma_{z}$ is not constant. As the stopping time decreases, the variation of the ratio becomes steep. Especially, those for $t<1 t_{\mathrm{K}}$ is prominent. In the model $t_{\text {stop }}=0.25$, the ratio $h / \sigma_{z}$ increases for $t<0.08 t_{\mathrm{K}}$. The maximum value is about 3.7 . The sound crossing time is $h / \sigma_{z} / \simeq 1.4$ in the gas-free model. When $\tilde{t}_{\text {stop }, 0}<1$, the dissipation is faster than the sound crossing. Therefore, the velocity dispersion decays before the scale height decays, and the ratio becomes larger than unity. In Figure 6, we can see the oscillation. The period is about $0.4 t_{\mathrm{K}}$, and the amplitude decreases gradually. The reason for the oscillation is not clear. This may be due to some kind of the relaxation process.

We cannot use the Roche density as the collapse criterion for $\tilde{t}_{\text {stop }}>1$. For $\tilde{t}_{\text {stop }, 0} \geq 4.0, Q_{R}$ is always larger than unity. But the large particles form. For $\tilde{t}_{\mathrm{stop}, 0}=2.0, Q_{R}$ becomes unity at $t / t_{\mathrm{K}}=2$, but the large particles start forming at $t / t_{\mathrm{K}}=0.6$. For $\tilde{t}_{\text {stop }} \leq 1$, the time when $Q_{R}=1$ corresponds to the time when the large particles start forming. When we derive the Roche criterion, we simply compare the self-gravity with the tidal force. However, we showed that the non-axisymmetric structure develops. The azimuthal motion may be important to discuss the formation of the wake-like structure. Therefore, the Roche criterion is not applicable.

The decay time of $Q_{R}$ value $t_{\text {decay,h }}$ is defined by

$$
t_{\text {decay }, \mathrm{h}}=t_{\text {min }, \mathrm{h}} \frac{h_{\text {init }}}{h_{\text {init }}-h_{\min }},
$$

where $h_{\text {init }}$ is the initial scale height of a dust layer. Figure 7 shows $t_{\text {decay,h }}$ as a function of $t_{\text {stop }, 0}$. The decay time of $Q_{R}$ has a minimum value at $\tilde{t}_{\mathrm{stop}, 0}=0.5$. For $\tilde{t}_{\mathrm{stop}, 0}<0.5$, the decay time shortens with the stopping time. For $\tilde{t}_{\text {stop }, 0}<0.5$, the strong coupled particles with gas fall to the midplane at the terminal velocity. The settling time is proportional to $\tilde{t}_{\text {stop }, 0}^{-1}$. On the other hand, for $\tilde{t}_{\text {stop }, 0}>0.5$, the decay time lengthens with longer stopping time. In this regime, the particles oscillate around the equatorial plane (Nakagawa et al. 1986). The decay time scale of the amplitude is proportional to the stopping time.

\subsubsection{Number of Planetesimal Seeds}

Figure 8 shows the time evolution of the number of planetesimal seeds per the area $\lambda_{\mathrm{m}}^{2}, N_{\mathrm{pl}}$. We define a particle whose mass is larger than $M_{\text {linear }}$ as a seed of planetesimals, where $M_{\text {linear }}$ is the planetesimal mass predicted by the linear theory $\pi \Sigma\left(\lambda_{\mathrm{m}} / 2\right)^{2}$. In all models, $N_{\mathrm{pl}}$ has a maximum value at $t / t_{\mathrm{K}}=0.5-1$. The number $N_{\mathrm{pl}}$ increases rapidly in the early stage, and decreases in the late stage. In the early stage, gravitational instability occurs and the small density fluctuation grows and many planetesimal seeds form. In this stage, the number of seeds increases. They form through the gravitational instability. Once many planetesimal seeds form, the mutual collisions 
among them start. The number of planetesimals decreases rapidly in the late stage. In the final state, almost all particles are absorbed by a few particles. The number of planetesimals in the final state depends on the size of the computational domain (paper II).

The peak of $N_{\mathrm{pl}}$ depends on the stopping time $\tilde{t}_{\mathrm{stop}, 0}$. Figure 9 shows the maximum number of the planetesimal $N_{\max }$ as a function of the stopping time $\tilde{t}_{\mathrm{stop}, 0}$. The maximum number of planetesimals $N_{\text {max }}$ has a peak at $\tilde{t}_{\text {stop }, 0} \simeq 0.5$. As discussed in 44.2 .2 , the scale height has the minimum value at $\tilde{t}_{\mathrm{stop}, 0} \simeq 0.5$. The characteristic length scale of gravitational instability corresponds to the scale height. As the characteristic length of gravitational instability shortens, the number of the planetesimals formed increases.

\subsubsection{Mass of Planetesimals}

Figure 10 shows the time evolution of the mass of the largest planetesimal. In all models, the largest planetesimal grows rapidly in $t / t_{\mathrm{K}}<5$, and the growth is stalled at $t / t_{\mathrm{K}} \simeq 5-6$. The growth in the gas-free model is slower than those in the gas models. As discussed in 4.2 .1 , the gas drag enhances the dissipation of the kinetic energy of dust. As the drag becomes stronger, the gravitational instability occurs earlier and the growth becomes more rapid.

The final mass of the largest planetesimal is $30-50 M_{\text {linear }}$. The clear dependence of the largest mass on the initial stopping time cannot be seen. As seeds grow, the stopping time lengthens. In the final state, we can neglect the effect of the drag. Therefore, the final mass and the number do not depend on the initial stopping time. Large planetesimals sweep small particles in the rotational direction. Therefore the final mass depends on the size of the computational domain (paper II).

\subsection{Effect of Rotational Velocity Difference and Drag Law}

The difference in rotational velocity between dust and gas causes the radial migration of dust. By neglecting the self-gravity and the time derivatives of Equation (18) and (19), we obtain the steady solution: (Adachi et al. 1976; Weidenschilling 1977)

$$
\tilde{v}_{x}=\frac{2 \tilde{t}_{\text {stop }} \tilde{v}_{\text {dif }}}{\tilde{t}_{\text {stop }}^{2}+1}
$$

Figure 11 shows the time evolution of the radial velocity $\tilde{v}_{x}$ of the largest particle. In these

models, the initial stopping time is $\tilde{t}_{\mathrm{stop}, 0}=1.0$. The power-law index and the velocity difference are $\left(\alpha, \tilde{v}_{\text {dif }}\right)=(0,10),(2 / 3,10),(0,20)$, and $(2 / 3,20)$ (models 100AC1, 100AS1, 100AC2, and 100AS2). In all models, the radial velocity $\tilde{v}_{x}$ is negative at $t / t_{\mathrm{K}}=1$. This corresponds to the radially inward drift. The rotational velocity of gas is slower than the Kepler velocity; dust experiences a headwind, which causes its inward migration. Therefore their radial velocities become negative. Although $\alpha$ 
is different, if $v_{\text {dif }}$ is same, the time evolutions of $v_{x}$ are similar in the early phase. This is because the planetesimals do not grow sufficiently in $t<1 t_{\mathrm{K}}$. If we estimate the terminal velocity from Equation (29) by the initial stopping time, they are the radial velocity $\tilde{v}_{\text {in }}=10$ for $\tilde{v}_{\text {dif }}=10$ and $\tilde{v}_{\text {in }}=20$ for $\tilde{v}_{\text {dif }}=20$. In models for $\alpha=0$, the drag coefficient of a particle is independent of its size, thus the radial velocity converges to $\tilde{v}_{x}=10$ for $\tilde{v}_{\text {dif }}=10$ and $\tilde{v}_{x}=20$ for $\tilde{v}_{\text {dif }}=20$; the radial migration does not stop. On the other hand, In models for $\alpha=2 / 3$, as planetesimals grow, the drag coefficient becomes small; the radial migration stops finally. The radial velocity oscillates with the period of about $t_{\mathrm{K}}$. This oscillation is caused by the epicycle motion. The gas drag is so weak that the oscillation is not damped.

\section{Discussion}

\subsection{Comparison with Linear Analyses}

Youdin (2005a) performed linear analyses of the dissipative gravitational instability of a dust layer. They studied the effect of the stopping time, the thickness of the dust layer, and the velocity dispersion. We summarized the essence of their results in $₫ 2$. The dust layer is always unstable for long wavelength modes. Thus, we should compare the timescale of the gravitational instability with those of other processes. Under the thin disk approximation $(h=0)$, we derived that simple condition that the gravitational instability dominates the other processes. Though the disk thickness cannot be neglected and the gravitational wakes is not axisymmetric, this condition corresponds to the occurrence of the gravitational wakes, as shown in 44 . To discuss the formation of the self-gravity wakes, Toomre's $Q$ value is more important than the Roche criterion $Q_{\mathrm{R}}$.

They applied the growth rate of the gravitational instability to the gravitational collapse in the turbulent disks. They assumed the simple turbulence model and studied the particle response to the turbulence flow. The protoplanetary disk can be turbulent due to magnetorotational instability or Kelvin-Helmholtz instability. They suggested that the standard hydrostatic relation $h \simeq \sqrt{2} \sigma_{z} / \Omega$ is not satisfied in the turbulent disk and the dust disk can be very thick. The thin disk approximation is clearly not valid. The criterion of the formation of the self-gravitational wakes may change. We plan to study the effect of the turbulence in the next paper.

\subsection{Comparison with Hydrodynamic Simulations}

Yamoto \& Sekiya (2006) performed the two-dimensional numerical simulation of the gravitational instability of a dust layer. They found that the dust layer becomes extremely thin if the stopping time is long, such as $\tilde{t}_{\text {stop }}>0.1$. This is because the dust settling is faster than the growth of the gravitational instability. However, our results show that the gravitational instability occurs although the stopping time is long. 
There are some differences between our simulations and theirs. We treated the dust component as particles. But, Yamoto \& Sekiva (2006) treated it as a pressure-free fluid. If the stopping time is short and the velocity dispersion is small, we can treat dust as a pressure-free fluid. The pressurefree fluid model becomes irrelevant for longer stopping times. For $\tilde{t}_{\text {stop }}=0.1$, we can use the pressure-free fluid, thus this should not cause the difference. In addition, the velocity dispersion stabilizes the gravitational instability generally. We neglected the dust back-reaction on the gas, but Yamoto \& Sekiva (2006) adopted two-component model and solved the interaction between them. If the dust density is high and the stopping time is short, the dust back-reaction on the gas should be important. The resulting gas flow may change the criterion. We performed threedimensional local calculation. On the other hand, Yamoto \& Sekiya (2006) assumed that the the dust layer to be axisymmetric with respect to the rotational axis. The gravitational instability forms the non-axisymmetric structures such as the gravitational wakes (e.g., Michikoshi et al. 2007; Wakita \& Sekiya 2008). The motion in the azimuthal direction is important to study the structure of the gravitationally unstable disk.

To understand the dynamics of planetesimal formation in gas, we need to perform threedimensional two-component simulations. We should consider the dust back-reaction on the gas and solve the hydrodynamic equations consistently. We will treat the dust component as particles because this method is applicable although the dust is loosely coupled to gas. We plan to study this point in the following papers of this series.

\section{Summary}

We performed local $N$-body simulations of the planetesimal formation through gravitational instability. In papers I and II, we adopted the gas-free model. However the gas is not negligible and is important to the formation of planetesimals. As a first step to understand the effect of gas, we considered gas as the background laminar flow. We neglected the dust back-reaction on the gas for the sake of simplicity. Laminar flow causes the dissipation of the kinetic energy and thus radial migration of dust.

In $\S 2$, we summarized the results of the linear analysis of the dissipative gravitational instability. To handle the dispersion relation analytically, we imposed the assumptions: the isothermal equation of state, the infinitely thin disk, and the axisymmetric perturbation. Simulations show that these assumptions are not valid, but they help us to understand the physical nature of the dissipative gravitational instability. We can use the growth rate to understand the simulation results. The long wavelength modes are always secularly unstable although $Q>1$. Therefore, we must compare with the timescale of the gravitational instability with other processes. We provided the equation (15) for the critical $Q$ value. If $Q$ is smaller than the critical value, the gravitational instability is a dominant process.

The numerical simulations show that the formation process of planetesimals is the same as that 
in the gas-free model. The formation process is divided into three stages qualitatively: the formation of wake-like structures, the creation of planetesimal seeds, and their collisional growth. By the linear stability analysis, the dust layer in the laminar gas disk is secularly unstable although $Q>1$. The growth time of the dissipative gravitational instability is slower than the dust sedimentation and the decrease of the velocity dispersion when the initial $Q$ value is sufficiently large. Thus, the disk shrinks vertically and $Q$ decreases. As the velocity dispersion and the scale height decrease, the growth rate of the gravitational instability increases. Finally, the gravitational instability becomes dominant. Then wake-like structures are formed by the gravitational instability. These structures fragment into planetesimal seeds. Seeds grow rapidly owing to the mutual collisions. Finally, almost all mass in the computational domain is absorbed by only one planetesimal in the calculation.

We investigated the dependence of results on the initial stopping time. The $Q$ value decreases in the initial stage, and it reaches the minimum. The decay time scale of $Q$ is proportional to the stopping time for $\tilde{t}_{\text {stop }, 0}<1$, which is the decay timescale of the velocity dispersion. The minimum $Q$ is determined by the balance between the dissipation and gravitational instability. At the minimum $\mathrm{Q}$, the wake-like structures are formed. The time evolution of the ratio of the dust layer density to the Roche density $Q_{R}$ is similar to that of $Q$. However the decay time scale of $Q_{R}$ is longer than that of $Q$. The time when $Q_{R}=1$ or $Q_{R}=Q_{R \text {,min }}$ does not correspond to the onset of the gravitational instability, such as the formation of the wake-like structures. This indicates that the Roche criterion is not applicable in this system. We investigated the time evolution of the number of planetesimal seeds. We define a particle whose mass is larger than $M_{\text {linear }}$ as a planetesimal seed, where $M_{\text {linear }}$ is the planetesimal mass predicted by the linear theory $\pi \Sigma\left(\lambda_{\mathrm{m}} / 2\right)^{2}$. The number of planetesimals has a maximum value. After the gravitational instability becomes a dominant process, planetesimal seeds form. In this stage, the number of planetesimal seeds increases. However, in the late stage, the number of seeds decreases by the mutual collisions among them. The maximum of the number of the planetesimal seeds depends on the initial stopping time. The maximum number of planetesimal seeds has a peak at $\tilde{t}_{\mathrm{stop}, 0} \simeq 0.5$. We are unable to identify a clear difference in the mass of the largest particle in the final state. The final mass of the planetesimal depends on the size of the computational domain (Michikoshi et al. 2009).

We also investigated the models where the gas rotational velocity is slower than the Kepler velocity. We confirmed that the planetesimal is able to form in the sub-Keplerian gas disk. In the initial stage, particles experience a headwind, which causes its inward migration. If we adopt Stokes' gas drag law, as planetesimals grow, the drag coefficient becomes small; the radial migration finally slows.

Almost all mass is absorbed by the largest planetesimal. In the gas-free models, we showed that the final mass of planetesimals depends on the size of computational domain. To investigate the final mass of planetesimals in detail, we should perform larger scale simulations. This remains to be discussed further. In addition, in this paper, we assumed gas to be laminar flow. However, the gas may be turbulent. If the turbulence is strong, the particles are stirred up and the gravitational instability may be prevented. The timescale for the dissipative gravitational instability is long 
(Ward 1976; Youdin 2005a). However, though the velocity dispersion of dust particles is large and the timescale for the instability is long, the dust layer may disrupt owing to the dissipative gravitational instability over time in the turbulent disk. In the next paper, we will investigate the particle response to the turbulence and the gravitational instability in the turbulent disk.

Numerical simulations were carried out on the MUV system at Center for Computational Astrophysics, National Astronomical Observatory Japan. This research was partially supported by MEXT (Ministry of Education, Culture, Sports, Science and Technology), Japan, the Grant-in-Aid for Scientific Research on Priority Areas, "Development of Extra-Solar Planetary Science," and the Special Coordination Fund for Promoting Science and Technology, "GRAPE-DR Project." S. I. is supported by Grants-in-Aid (16077202, and 18540238) from MEXT of Japan.

\section{REFERENCES}

Adachi, I., Hayashi, C., \& Nakazawa, K. 1976, Progress of Theoretical Physics, 56, 1756

Balbus, S. A. \& Hawley, J. F. 1991, ApJ, 376, 214

Barge, P. \& Sommeria, J. 1995, A\&A, 295, L1

Barranco, J. A. 2009, ApJ, 691, 907

Blum, J. \& Wurm, G. 2000, Icarus, 143, 138

Chiang, E. 2008, ApJ, 675, 1549

Chiang, E. \& Youdin, A. N. 2010, Annual Review of Earth and Planetary Sciences, 38, 493

Coradini, A., Magni, G., \& Federico, C. 1981, A\&A, 98, 173

Cuzzi, J. N., Dobrovolskis, A. R., \& Champney, J. M. 1993, Icarus, 106, 102

Daisaka, H. \& Ida, S. 1999, Earth, Planets, and Space, 51, 1195

Dobrovolskis, A. R., Dacles-Mariani, J. S., \& Cuzzi, J. N. 1999, J. Geophys. Res., 104, 30805

Fromang, S. \& Nelson, R. P. 2006, A\&A, 457, 343

Goldreich, P. \& Tremaine, S. D. 1978, Icarus, 34, 227

Goldreich, P. \& Ward, W. R. 1973, ApJ, 183, 1051

Gómez, G. C. \& Ostriker, E. C. 2005, ApJ, 630, 1093

Goodman, J. \& Pindor, B. 2000, Icarus, 148, 537 
Ida, S. \& Makino, J. 1992, Icarus, 96, 107

Inutsuka, S. \& Sano, T. 2005, ApJ, 628, L155

Ishitsu, N. \& Sekiya, M. 2002, Earth, Planets, and Space, 54, 917

—. 2003, Icarus, 165, 181

Johansen, A., Henning, T., \& Klahr, H. 2006, ApJ, 643, 1219

Johansen, A., Oishi, J. S., Low, M.-M. M., Klahr, H., Henning, T., \& Youdin, A. 2007, Nature, 448,1022

Johansen, A. \& Youdin, A. 2007, ApJ, 662, 627

Johansen, A., Youdin, A., \& Mac Low, M. 2009, ApJ, 704, L75

Kokubo, E., Ida, S., \& Makino, J. 2000, Icarus, 148, 419

Landau, L. D. \& Lifshitz, E. M. 1959, Fluid mechanics

Michikoshi, S. \& Inutsuka, S.-i. 2006, ApJ, 641, 1131

Michikoshi, S., Inutsuka, S.-i., Kokubo, E., \& Furuya, I. 2007, ApJ, 657, 521

Michikoshi, S., Kokubo, E., \& Inutsuka, S.-i. 2009, ApJ, 703, 1363

Nakagawa, Y., Sekiya, M., \& Hayashi, C. 1986, Icarus, 67, 375

Nakazawa, K. \& Ida, S. 1988, Progress of Theoretical Physics Supplement, 96, 167

Petit, J.-M. \& Henon, M. 1986, Icarus, 66, 536

Rein, H., Lesur, G., \& Leinhardt, Z. M. 2010, A\&A, 511, A69+

Richardson, D. C. 1994, MNRAS, 269, 493

Safronov, V. 1969, Evolution of the Protoplanetary Cloud and Formation of the Earth and Planets (NASA Tech. Trans. TT-F-677; Moscow)

Salo, H. 1995, Icarus, 117, 287

Sano, T., Miyama, S. M., Umebayashi, T., \& Nakano, T. 2000, ApJ, 543, 486

Sekiya, M. 1983, Progress of Theoretical Physics, 69, 1116

-. 1998, Icarus, 133, 298

Sekiya, M. \& Ishitsu, N. 2000, Earth, Planets, and Space, 52, 517 
-. 2001, Earth, Planets, and Space, 53, 761

Sirono, S.-I. 2004, Icarus, 167, 431

Tanga, P., Weidenschilling, S. J., Michel, P., \& Richardson, D. C. 2004, A\&A, 427, 1105

Toomre, A. 1964, ApJ, 139, 1217

Vandervoort, P. O. 1970, ApJ, 161, 87

Wakita, S. \& Sekiya, M. 2008, ApJ, 675, 1559

Ward, W. R. 1976, in Frontiers of Astrophysics, 1-40

Weidenschilling, S. J. 1977, MNRAS, 180, 57

—. 1980, Icarus, 44, 172

Weidenschilling, S. J. \& Cuzzi, J. N. 1993, in Protostars and Planets III, ed. E. H. Levy \& J. I. Lunine, $1031-1060$

Wisdom, J. \& Tremaine, S. 1988, AJ, 95, 925

Yamoto, F. \& Sekiya, M. 2004, Icarus, 170, 180

-. 2006, ApJ, 646, L155

Youdin, A. \& Johansen, A. 2007, ApJ, 662, 613

Youdin, A. N. 2005a, ArXiv Astrophysics e-prints, astro-ph/0508659

—. 2005b, ArXiv Astrophysics e-prints, astro-ph/0508659

Youdin, A. N. \& Goodman, J. 2005, ApJ, 620, 459

This preprint was prepared with the AAS LATEX macros v5.2. 

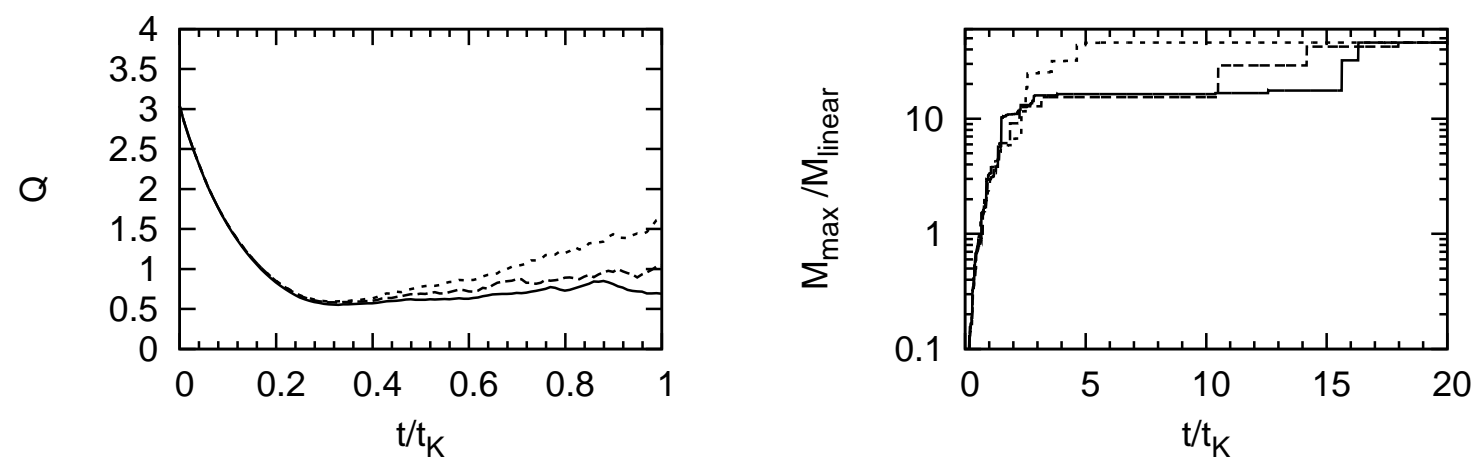

Fig. 2.- The time evolutions of Toomre's $Q$ value (left panel) and the maximum mass $M_{\max }$ of planetesimals (right panel) for hard-sphere model (solid curve), accretion model for $\alpha=0$ (dashed curve), and accretion model for $\alpha=2 / 3$ (short dashed curve). 

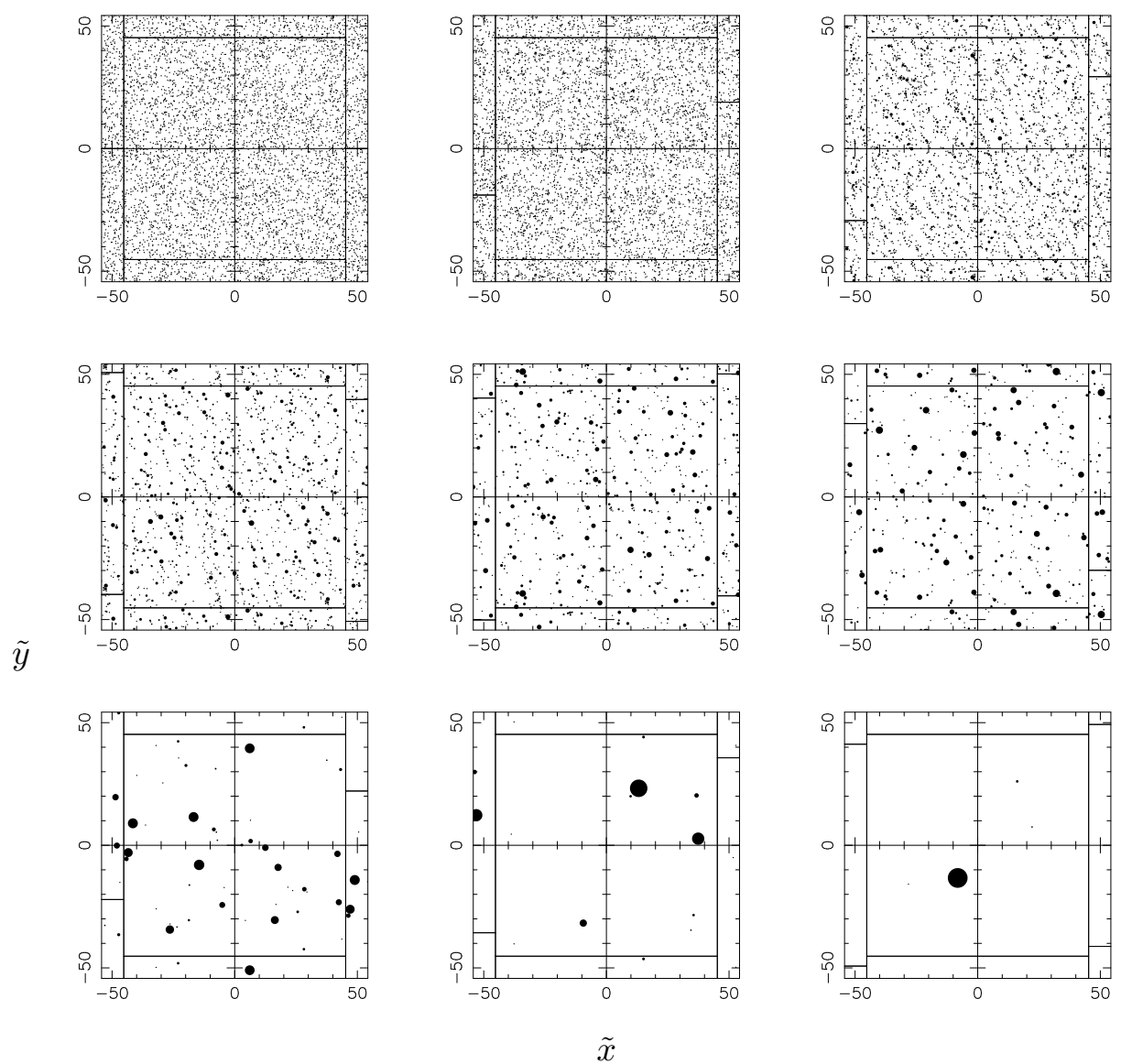

Fig. 3.- Spatial distribution of particles in the $\tilde{x} \tilde{y}$ plane in in the model 100 AS0 at $t / t_{\mathrm{K}}=$ 0.0, 0.2, 0.4, 0.6, 0.8, 1.0, 2.0, 4.0, and 6.0 from left to right and from top to bottom. Circles represent particles and their size is proportional to the physical size of particles. 


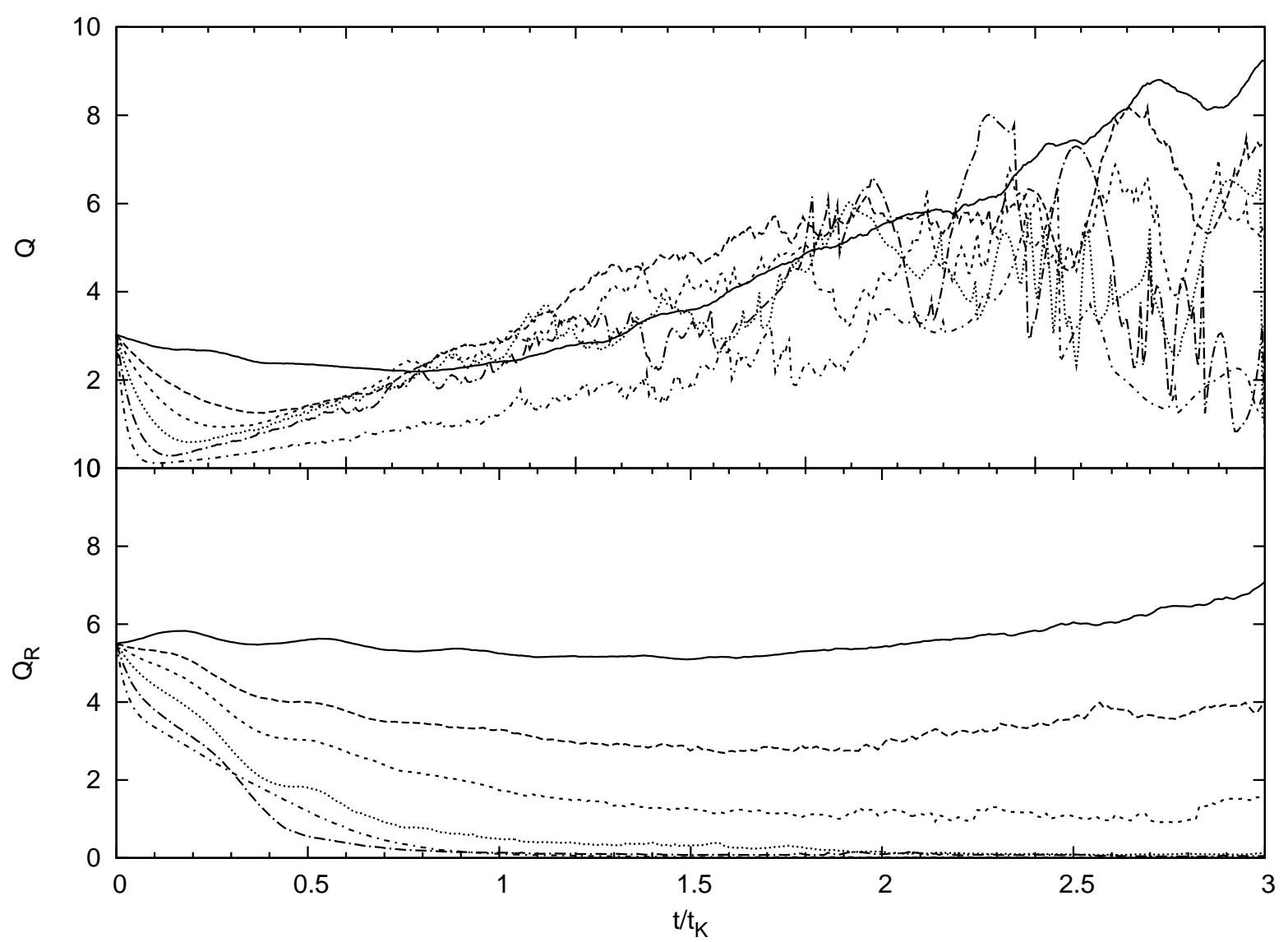

Fig. 4.- The time evolutions of Toomre's $Q$ value (top panel) and the normalized scale height (bottom panel) for gas-free model (solid curve), $\tilde{t}_{\text {stop }, 0}=4.0$ (dashed curve), $\tilde{t}_{\text {stop }, 0}=2.0$ (short dashed curve), $\tilde{t}_{\mathrm{stop}, 0}=1.0$ (dotted curve), $\tilde{t}_{\mathrm{stop}, 0}=1.0$ (dash-dotted curve), and $\tilde{t}_{\mathrm{stop}, 0}=0.5$ (dot-short-dashed curve) (models INFA, 400AS0, 200AS0, 100AS0, 050AS0, and 025AS0). 


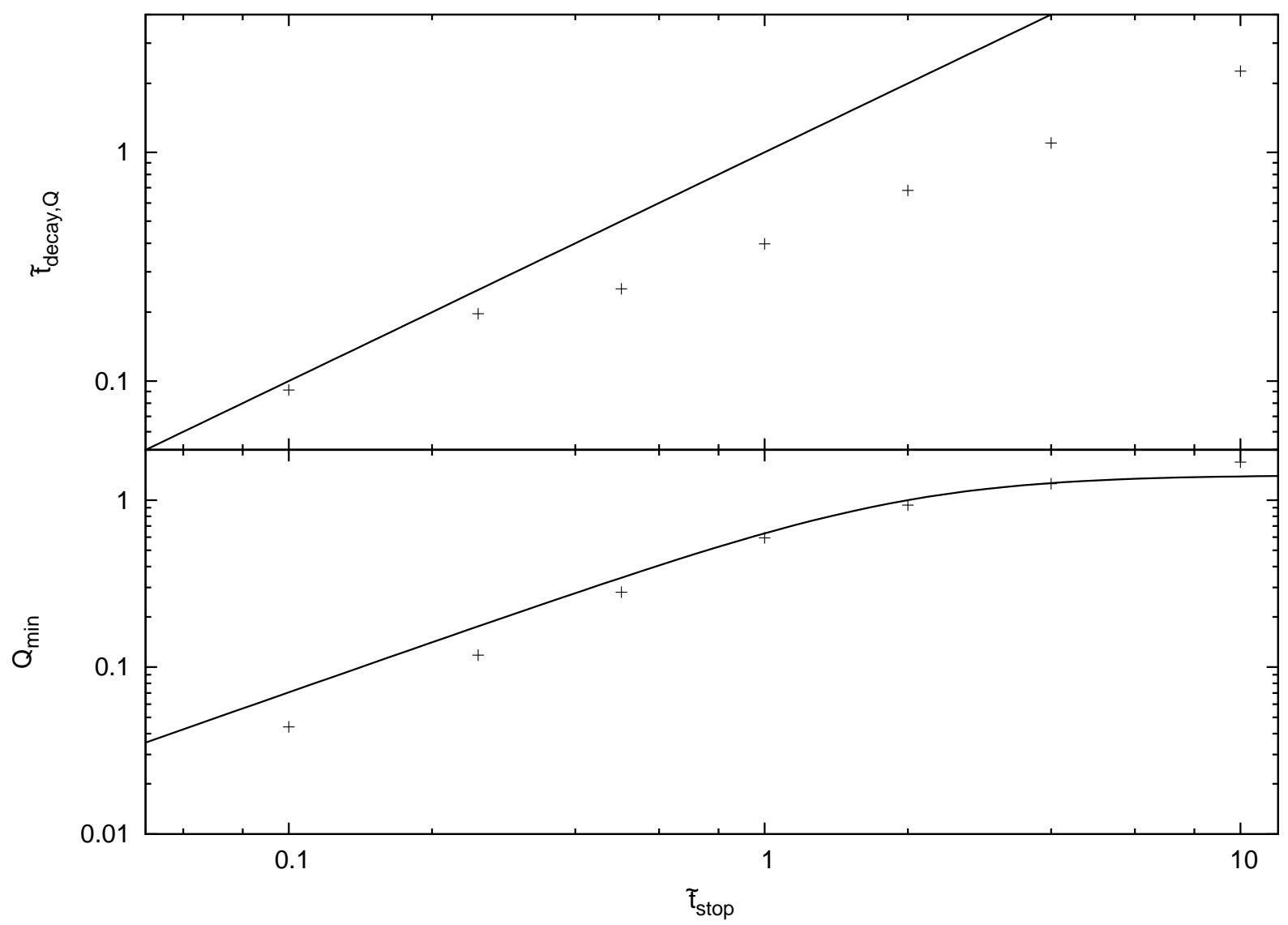

Fig. 5. - The decay time $\tilde{t}_{\text {decay }, \mathrm{Q}}$ of the Toomre's $Q$ value (top panel) and the minimum $Q$ value $Q_{\min }$ (bottom panel) as a function of $\tilde{t}_{\text {stop }, 0}$. The cross denotes the result of Numerical simulations (models 010AS0, 025AS0, 050AS0, 100AS0, 200AS0, 400AS0, and 1000AS0). In the top panel, the solid line shows the line $\tilde{t}_{\text {decay, },}=\tilde{t}_{\text {stop }, 0}$. In the bottom panel, the solid line shows the analytical estimation of the minimum Toomre's $Q$ value expressed by Equation (25). 


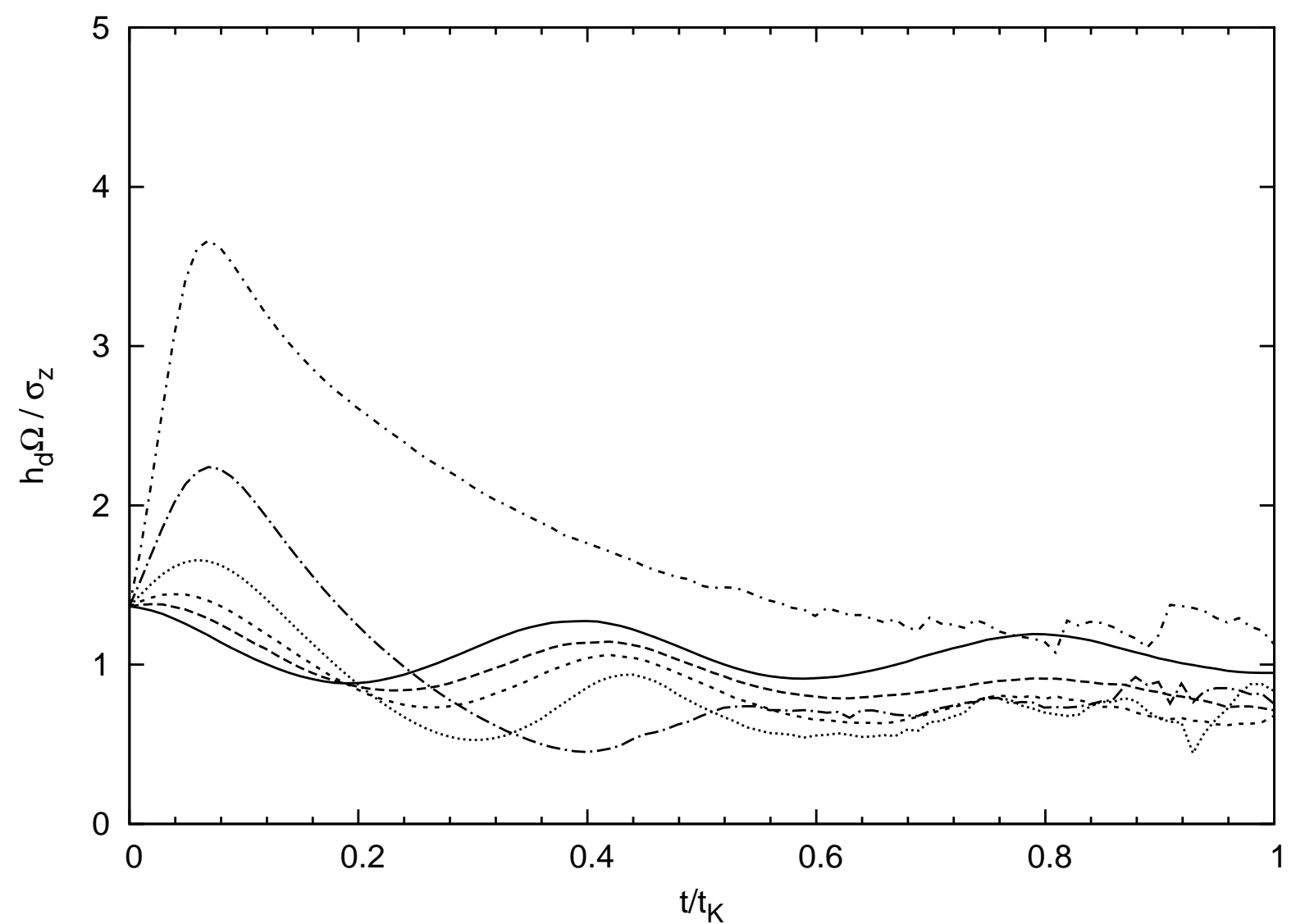

Fig. 6. - The time evolutions of $\tilde{h} / \tilde{\sigma}$ for gas-free model (solid curve), $\tilde{t}_{\text {stop }, 0}=4.0$ (dashed curve), $\tilde{t}_{\text {stop }, 0}=2.0$ (short dashed curve),$\tilde{t}_{\text {stop }, 0}=1.0$ (dotted curve), $\tilde{t}_{\text {stop }, 0}=0.5$ (dash-dotted curve), and $\tilde{t}_{\text {stop }, 0}=0.25$ (dot-short-dashed curve) (models INFA, 400AS0, 200AS0, 100AS0, 050AS0, and 025AS0). 


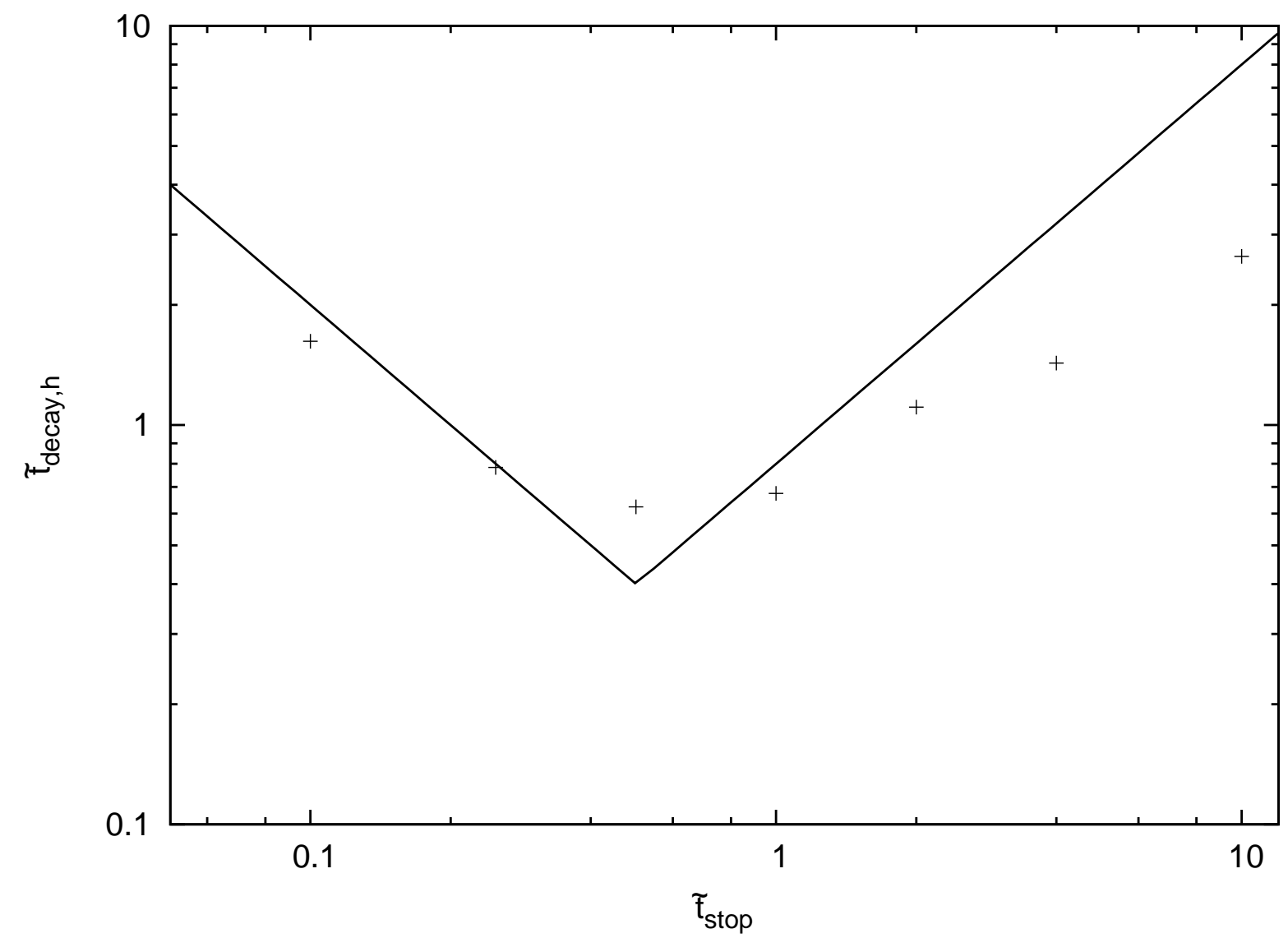

Fig. 7.- The decay time of the scale height as a function of $\tilde{t}_{\mathrm{stop}, 0}$. The cross denotes the result of Numerical simulations (models 010AS0, 025AS0, 050AS0, 100AS0, 200AS0, 400AS0, and 1000AS0). 


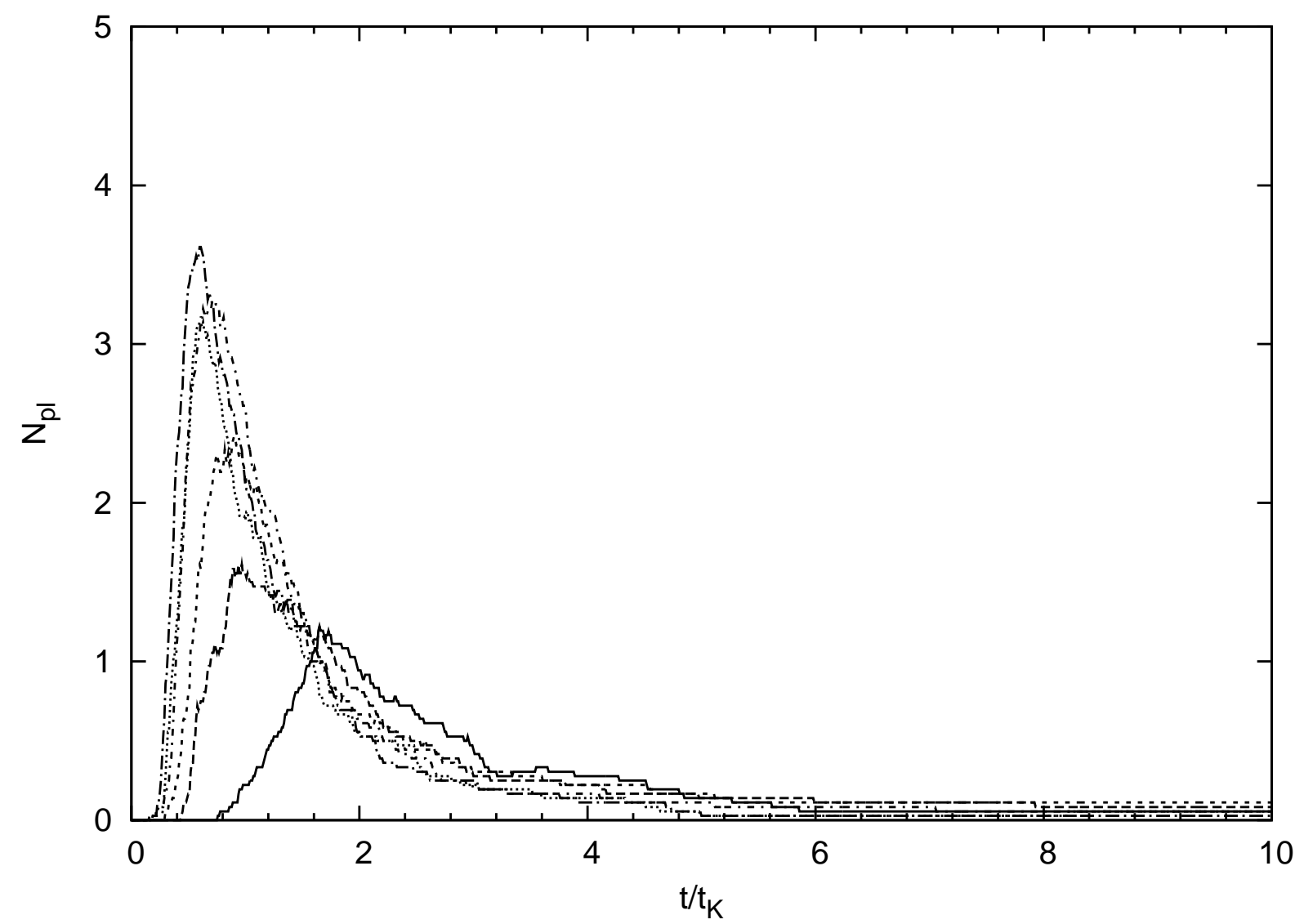

Fig. 8. - The time evolutions of the number of the planetesimals for gas-free model (solid curve), $\tilde{t}_{\text {stop }, 0}=4.0$ (dashed curve), $\tilde{t}_{\text {stop }, 0}=2.0$ (short dashed curve), $\tilde{t}_{\text {stop }, 0}=1.0$ (dotted curve) , $\tilde{t}_{\text {stop }, 0}=0.5$ (dash-dotted curve), and $\tilde{t}_{\text {stop }, 0}=0.25$ (dot-short-dashed curve) (models INFA, 400AS0, 200AS0, 100AS0, 050AS0, and 025AS0). 


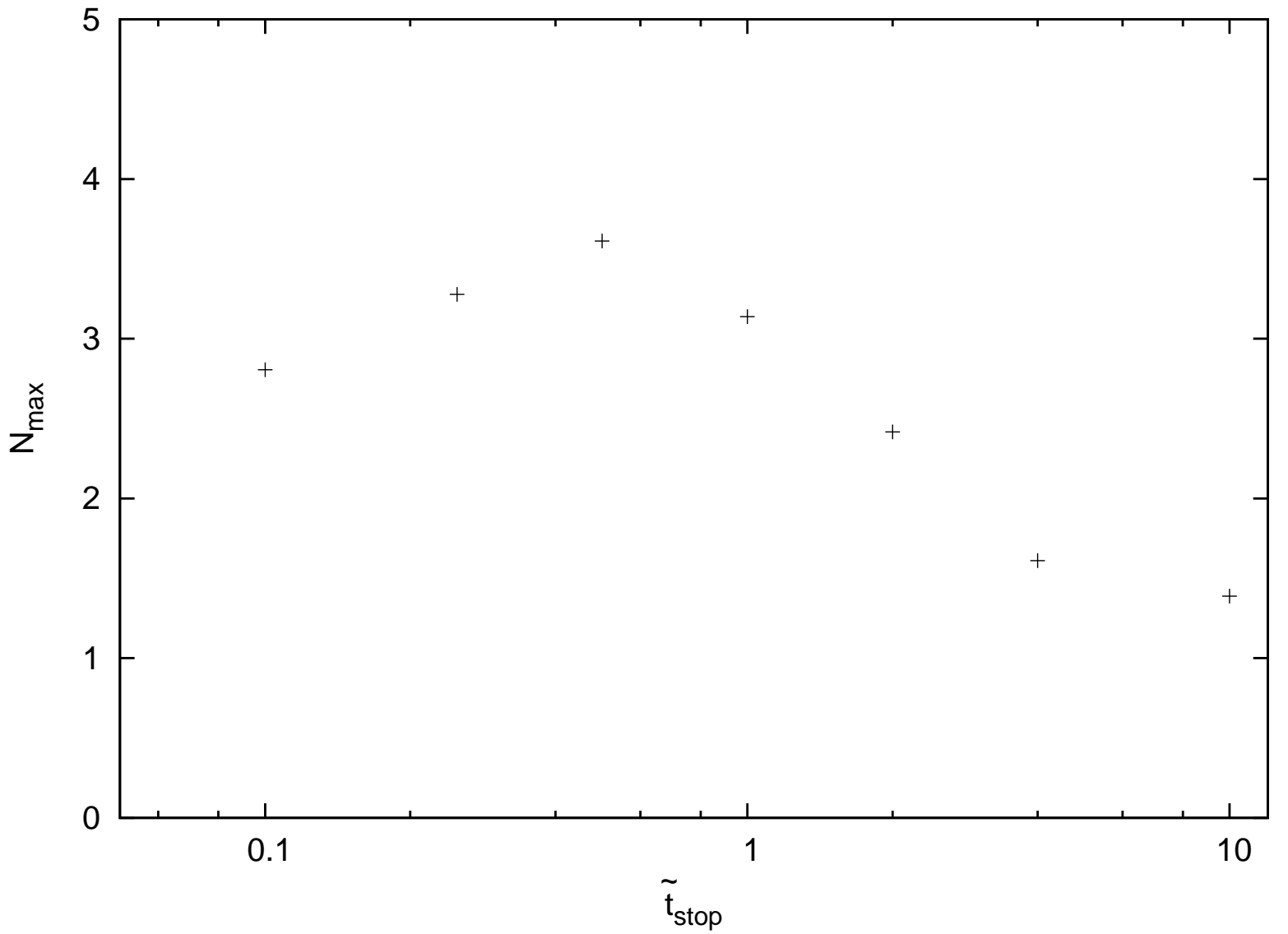

Fig. 9. - The maximum number of the planetesimals as a function of $\tilde{t}_{\mathrm{stop}, 0}$. The cross denotes the result of Numerical simulations (models 010AS0, 025AS0, 050AS0, 100AS0, 200AS0, 400AS0, and 1000AS0). 


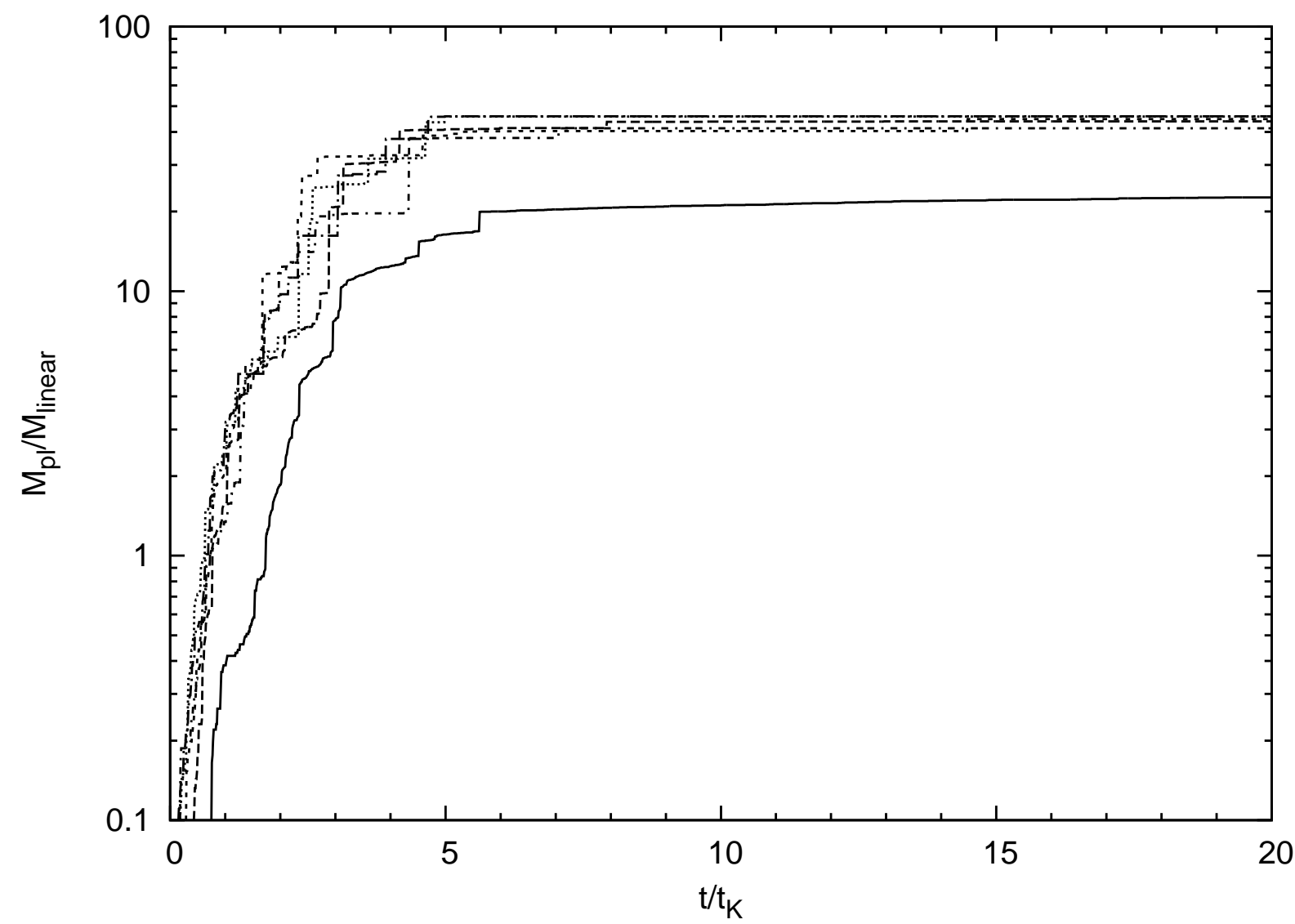

Fig. 10.- The time evolutions of the mass of the largest planetesimal for gas-free model (solid curve), $\tilde{t}_{\mathrm{stop}, 0}=4.0$ (dashed curve), $\tilde{t}_{\mathrm{stop}, 0}=2.0$ (short dashed curve), $\tilde{t}_{\mathrm{stop}, 0}=1.0$ (dotted curve), $\tilde{t}_{\text {stop }, 0}=0.5$ (dash-dotted curve), and $\tilde{t}_{\text {stop }, 0}=0.25$ (dot-short-dashed curve) (models INFA, 400AS0, 200AS0, 100AS0, 050AS0, and 025AS0). 


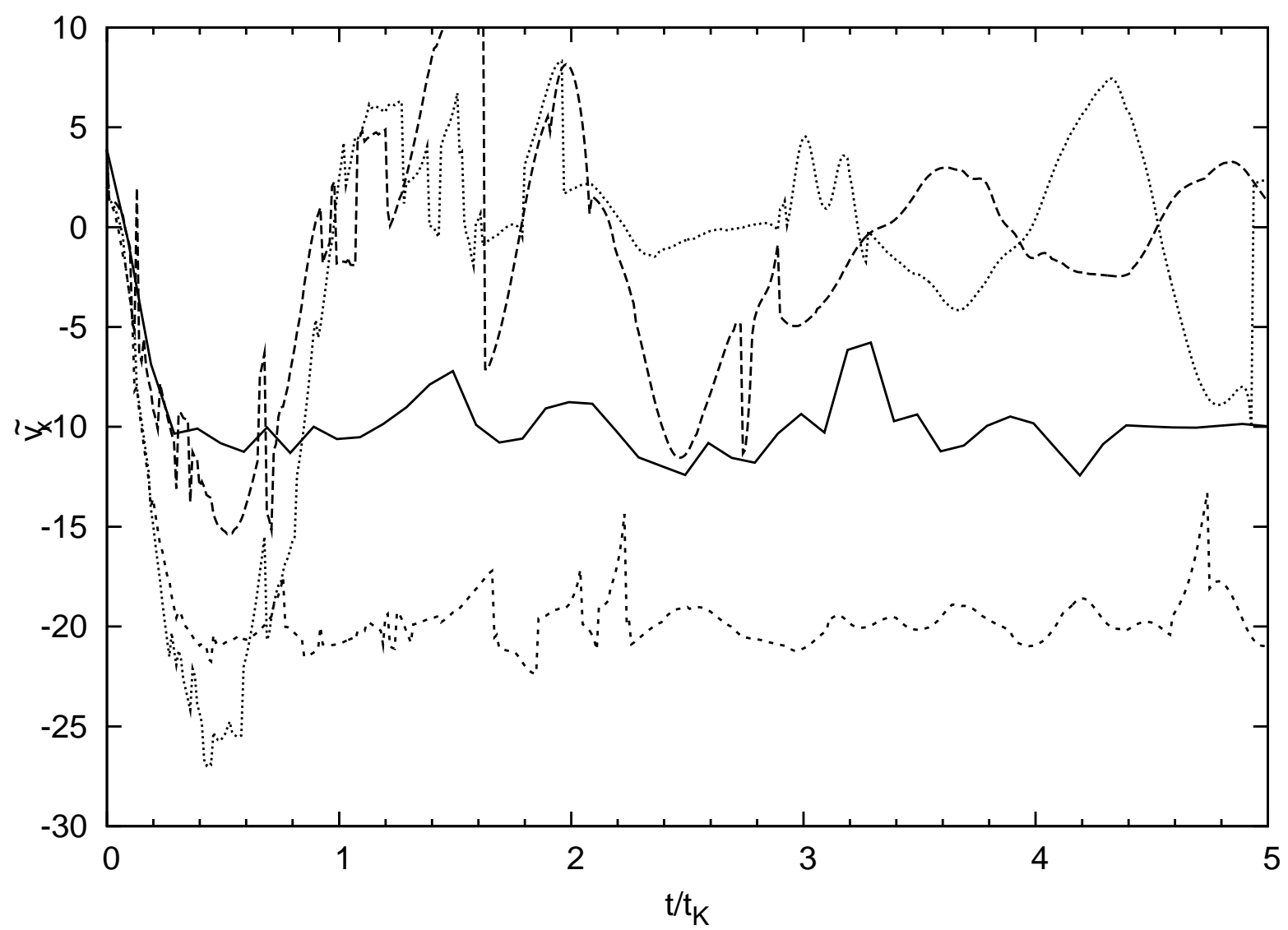

Fig. 11. - The time evolution of the radial velocity $v_{x}$ of the largest planetesimal for models $100 \mathrm{AC} 1$ (solid curve), 100AS1 (dashed curve), 100AC2 (short dashed curve), and 100AS2 (dotted curve). Note that the planetesimal whose velocity is plotted is not identical, i.e., the largest planetesimal changes as the other planetesimal grows. Therefore the velocity changes abruptly then. 